\title{
Homoserine Lactones Influence the Reaction of Plants to Rhizobia
}

Azhar A. Zarkani ${ }^{1, \dagger}$, Elke Stein ${ }^{1}$, Christian R. Röhrich ${ }^{2}$, Marek Schikora ${ }^{3}$, Elena Evguenieva-Hackenberg ${ }^{4}$, Thomas Degenkolb ${ }^{1}$, Andreas Vilcinskas ${ }^{1,2}$, Gabriele Klug ${ }^{4}$, Karl-Heinz Kogel ${ }^{1}$ and Adam Schikora ${ }^{1, *}$

1 Institute of Phytopathology and Applied Zoology, Centre for BioSystems, Land Use and Nutrition, Justus Liebig University Giessen, Heinrich-Buff-Ring 26-32, D-35392 Giessen, Germany; E-Mails: azhar.a.zarkani@agrar.uni-giessen.de (A.A.Z.); elke.stein@agrar.uni-giessen.de (E.S.); thomas.degenkolb@ernaehrung.uni-giessen.de (T.D.); andreas.vilcinskas@agrar.uni-giessen.de (A.V.); karl-heinz.kogel@agrar.uni-giessen.de (K.-H.K.)

2 Fraunhofer Institute for Molecular Biology and Applied Ecology (IME), Bioresources Project Group, Winchesterstrasse 2, D-35394 Giessen, Germany; E-Mail: christian.r.roehrich@fhg.uni-giessen.de

3 Department Sensor Data and Information Fusion, Fraunhofer FKIE, 53343 Wachtberg, Germany; E-Mail: marek.schikora@fkie.fraunhofer.de

4 Institute of Microbiology and Molecular Biology, Centre for BioSystems, Land Use and Nutrition, Justus Liebig University Giessen, Heinrich-Buff-Ring 26-32, D-35392 Giessen, Germany; E-Mails: elena.evguenieva-hackenberg@mikro.bio.uni-giessen.de (E.E.-H.); gabriele.klug@mikro.bio.uni-giessen.de (G.K.)

$\dagger$ Present address: Biotechnology Department, College of Science, University of Baghdad, Iraq.

* Author to whom correspondence should be addressed;

E-Mail: adam.schikora@agrar.uni-giessen.de; Tel.: +49-641-99-37497; Fax: +49-641-99-37499.

Received: 2 July 2013; in revised form: 8 August 2013 / Accepted: 12 August 2013 /

Published: 20 August 2013

Abstract: Bacterial quorum sensing molecules not only grant the communication within bacterial communities, but also influence eukaryotic hosts. $\mathrm{N}$-acyl-homoserine lactones (AHLs) produced by pathogenic or beneficial bacteria were shown to induce diverse reactions in animals and plants. In plants, the reaction to AHLs depends on the length of the lipid side chain. Here we investigated the impact of two bacteria on Arabidopsis thaliana, which usually enter a close symbiosis with plants from the Fabaceae 
(legumes) family and produce a long-chain AHL (Sinorhizobium meliloti) or a short-chain AHL (Rhizobium etli). We demonstrate that, similarly to the reaction to pure AHL molecules, the impact, which the inoculation with rhizosphere bacteria has on plants, depends on the type of the produced AHL. The inoculation with oxo-C14-HSL-producing $S$. meliloti strains enhanced plant resistance towards pathogenic bacteria, whereas the inoculation with an AttM lactonase-expressing S. meliloti strain did not. Inoculation with the oxo-C8-HSL-producing $R$. etli had no impact on the resistance, which is in agreement with our previous hypothesis. In addition, plants seem to influence the availability of AHLs in the rhizosphere. Taken together, this report provides new insights in the role of $\mathrm{N}$-acyl-homoserine lactones in the inter-kingdom communication at the root surface.

Keywords: quorum sensing; induced resistance; plant-bacteria interaction; homoserine lactones

\section{Introduction}

Many Gram-negative bacteria use $N$-acyl-homoserine lactones (AHLs) for their intra-population communication. Such communication between bacterial individuals, termed quorum sensing (QS), was discovered more than 40 years ago [1,2]. Today, we understand very well the mechanisms of QS within the bacterial populations. Notably, QS molecules used by beneficial or pathogenic bacteria have also impact on the eukaryotic host. The first indication that QS-molecules of rhizosphere bacteria influence plant defense responses came from a study on the interaction between Serratia liquefaciens MG1 and tomato (Solanum lycopersicum) [3]. Serratia liquefaciens MG1 produces C4- and C6-homoserine lactones when colonizing the root surface [4]. Colonization of the root surface with S. liquefaciens induced systemic resistance against the leaf-pathogenic fungus Alternaria alternata in tomato, whereas the AHL-negative $S$. liquefaciens mutant MG44 was not able to induce such resistance [3]. In a similar manner, colonization with the AHL-producing Serratia plymuthica wild-type strain HRO-C48 protected cucumber plants (Cucumis sativus) from the damping-off disease caused by the oomycete Pythium aphanidermatum, as well as tomato and bean (Phaseolus vulgaris) from infection with Botrytis cinerea, the causing agent of gray mold [5]. Similar to the study with S. liquefaciens MG44 and tomato, the authors demonstrated that the splI mutant of S. plymuthica, impaired in the production of AHLs, could not provide protection against $P$. aphanidermatum and B. cinerea. These results provide evidence that AHLs play an important role in the induction of plant defences. However, contradictory results were reported for the interaction between Arabidopsis thaliana and S. liquefaciens MG1 and its AHL-negative mutant MG44 [6]. Because the resistance against the pathogenic bacterium Pseudomonas syringae on $A$. thaliana leaves was not differently induced by the $S$. liquefaciens wild type and its AHL-negative mutant, the authors suggested an AHL-independent resistance increasing effect against $P$. syringae caused by root colonization with $S$. liquefaciens [6]. Thus, bacteria-plant interaction experiments with living microbial cells have to be interpreted very carefully, since different bacteria can induce different plant responses 
independently of the QS auto-inducer system. A helpful mean to circumvent this disturbing overlap effect was the use of pure AHL-compounds.

AHLs vary in the length of the lipid side chain and the substitution on the $\mathrm{C}_{3}$-atom $(\mathrm{O}$ - or OH-group). The length of the lipid side chain is important for the reaction of plants. C4-HSL, C6-HSL, oxo-C6-HSL and oxo-C8-HSL promoted growth of Arabidopsis [6-8]. Oxo-C10-HSL induced the formation of adventitious roots in mung beans [9]. On the other hand, oxo-C14-HSL and to a lesser extend OH-C14-HSL induced resistance in Arabidopsis and barley plants towards biotrophic and hemibiotrophic pathogens [10]. Likewise, oxo-C12-HSL has a resistance-inducing potential, though weaker than C14-HSL derivatives [8]. Comparison of five different AHLs differing in the length of their lipid side chain, which ranged from 6 to 14 carbons, on plant growth revealed clear differences.

We here report the effect of long-chain oxo-C14-HSL-producing Sinorhizobium meliloti on plant resistance. We show that the effect is dependent on the presence and type of the AHL, since only the inoculation with $S$. meliloti strains producing oxo-C14-HSL had a positive effect on Arabidopsis resistance towards pathogenic bacteria. In contrast, inoculation with an AHL-negative $S$. meliloti strain or an oxo-C8-HSL-producing Rhizobium etli was not able to enhance resistance. Remarkably, the impact of AHLs on plant resistance is independent of the native host-symbiont system, as shown by the influence on Arabidopsis, which is not forming nodules and not a symbiotic host for both $S$. meliloti and $R$. etli. In addition, our results suggest that plants influence the amount of QS molecules in the rhizosphere and therefore might interfere with the bacterial intercellular communication.

\section{Results}

\subsection{S. meliloti Rm2011 Produces N-3-Oxo-Tetradecanoyl-L-Homoserine Lactone (oxo-C14-HSL)}

In our previous report we showed that long-chain AHLs (e.g., oxo-C14-HSL) induce resistance in A. thaliana towards hemibiotrophic and biotrophic pathogens [10]. S. meliloti produces different long-chain AHLs [11] and was therefore a good candidate to study the interaction between A. thaliana and long-chain AHLs produced by rhizosphere bacteria. To commence this study, we determined which AHLs are synthesized in our conditions by $S$. meliloti $\mathrm{Rm} 2011$, a natural mutant with insertion in one of the AHL receptor genes, expR [12]. In order to define the type of AHLs, we used an LC-MS/MS approach. Bacteria were grown in $80 \mathrm{ml}$ of TY medium [13] and the cell-free culture supernatant was used for the analysis. As standards, we used commercially available C6-, oxo-C8-, oxo-C10-, oxo-C12-, and oxo-C14-HSL. We detected oxo-C14-HSL (Figure 1), confirming the previous results with other $S$. meliloti strains including the closely related strain Rm1021, which is also an $\operatorname{expR}$ mutant [12]. The identity of oxo-C14-HSL was confirmed by the presence of two pseudomolecular ions $\mathrm{m} / z 326.2\left([\mathrm{M}+\mathrm{H}]^{+}\right)$and $348.2\left([\mathrm{M}+\mathrm{Na}]^{+}\right)$. The pseudomolecular ion 326.2 $\left([\mathrm{M}+\mathrm{H}]^{+}\right)$was subsequently selected as a precursor for unambiguous HR-MS/MS identification $m / z$ : $326.2327\left(\mathrm{C}_{12} \mathrm{H}_{19} \mathrm{NO}_{4}, \quad[\mathrm{M}+\mathrm{H}]^{+}\right), \quad 225.1819 \quad\left(\mathrm{C}_{14} \mathrm{H}_{25} \mathrm{O}_{2}{ }^{+}\right), \quad 183.1717 \quad\left(\mathrm{C}_{12} \mathrm{H}_{23} \mathrm{O}^{+}\right)$, $102.0524\left(\mathrm{C}_{4} \mathrm{H}_{8} \mathrm{NO}_{2}^{+}\right), 74.0591\left(\mathrm{C}_{3} \mathrm{H}_{8} \mathrm{NO}^{+}\right), 56.0492\left(\mathrm{C}_{3} \mathrm{H}_{6} \mathrm{~N}^{+}\right)$. All diagnostic adduct and fragment ions listed above were confirmed by analyzing the reference standard. 
Figure 1. Verification of $N$-acyl-homoserine lactone (AHL) production in S. meliloti Rm2011. HPLC samples were screened in the positive ion mode from $\mathrm{m} / \mathrm{z} 50$ to 400. (A) The MS of standard oxo-C14-HSL standard $\left(\mathrm{m} / \mathrm{z}=326.2[\mathrm{M}+\mathrm{H}]^{+}\right)$shows a pattern similar to the AHL extracted from $S$. meliloti Rm2011 culture; (B) Further analysis of $m / z 326.2$ by MS/MS confirmed the identity of AHL extracted from S. meliloti Rm2011 culture with the standard oxo-C14-HSL. The diagnostic fragment ions $\mathrm{m} / z 102.0524$ and 102.0537 are indicative for the presence of the lactone ring. The highlighted $\mathrm{m} / \mathrm{z}$ values represent diagnostic fragment ions. Diamonds mark the pseudomolecular ions used as precursors for MS/MS analysis.

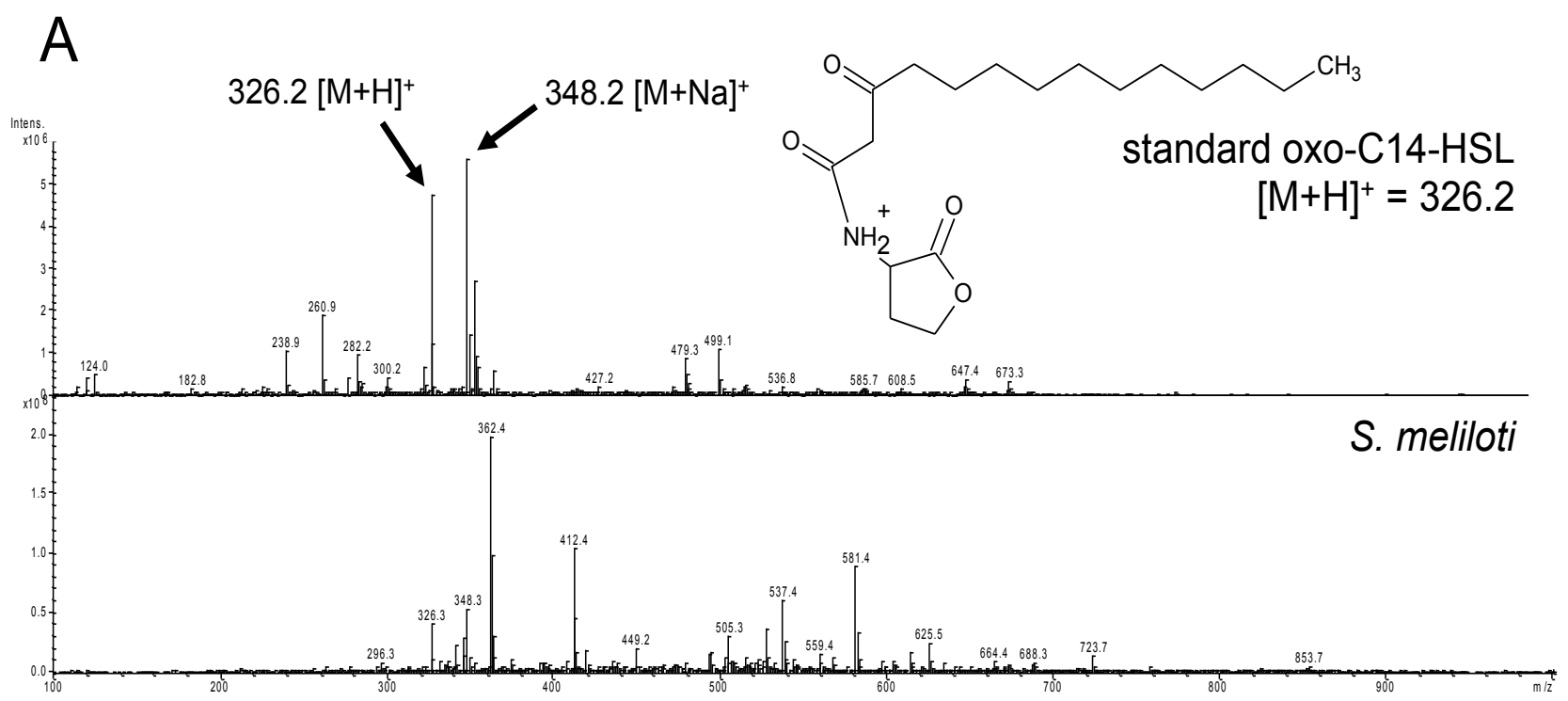

B

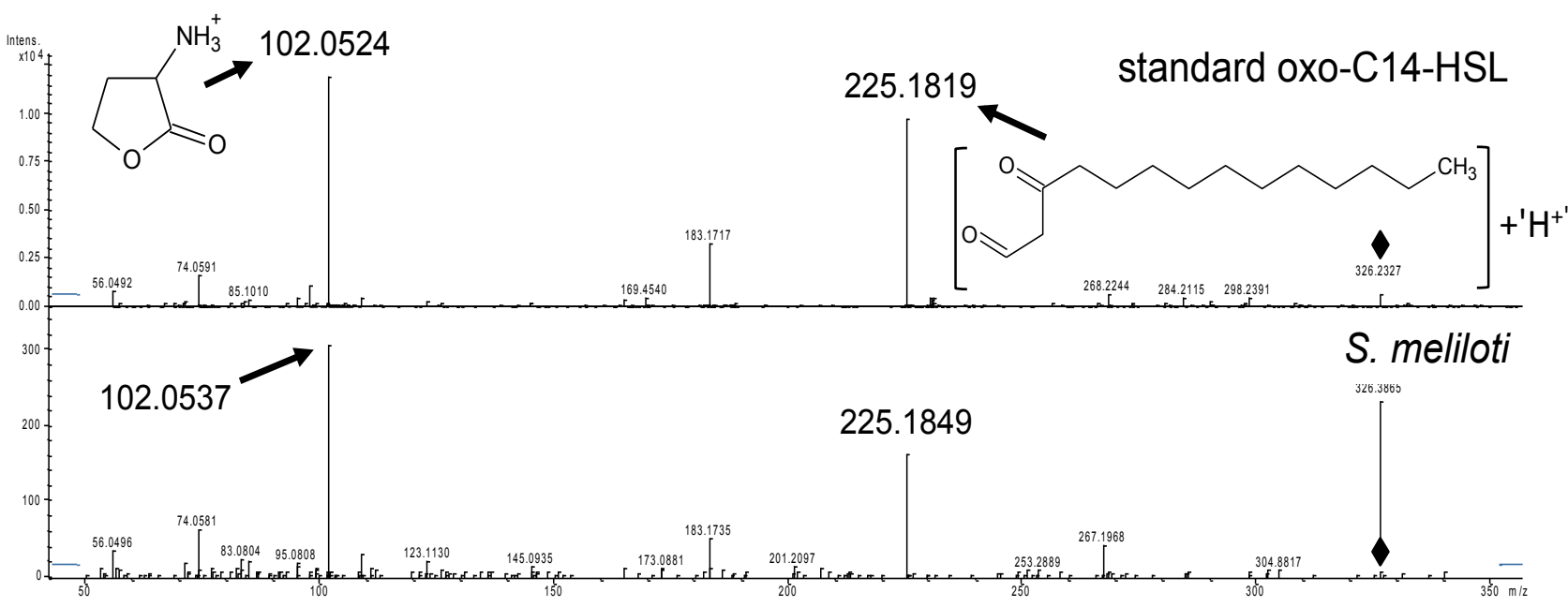

\subsection{Expression of the AttM Lactonase Abolishes AHL Accumulation in S. meliloti}

Since the eventual aim of this study was to investigate the AHL-dependent interaction between bacteria and plants, we aimed at an AHL-negative strain of $S$. meliloti, which could be used as a control in our study. The Agrobacterium tumefaciens attM gene encoding a lactonase, an enzyme 
hydrolyzing the lactone ring of an AHL molecule, was chosen for expression in S. meliloti in order to achieve this purpose. After initial check of resistance (data not shown), the kanamycin-resistance conferring, broad host range plasmid pBBR2-attM was constructed and introduced into the E. coli S17-1 strain [14] used subsequently for conjugation with $S$. meliloti Rm2011. The resulting AHL-negative strain was tested for AHL accumulation using the bacterial biosensor strain Pseudomonas putida KS35 which expresses lasB::gfp in an AHL-dependent manner [14]. S. meliloti was grown until $\mathrm{OD}_{600 \mathrm{~nm}}=1.3$, and AHLs were extracted from culture filtrate. Extracted AHLs were redissolved in $\mathrm{Me}_{2} \mathrm{CO}$ (acetone) and applied onto lawns of biosensor bacteria, which recognize oxo-C14-HSL (Figure S1). S. meliloti Rm2011 (pBBR2-attM) called hereafter att $^{+}$, showed no detectable AHL accumulation (Figure 2A). In addition, we tested how the lack of AHLs influences the growth of rhizobia. The $S$. meliloti attM strain, unable to produce a detectable amount of oxo-C14-HSL, shows delayed growth if compared to the reference strains (S. meliloti Rm2011, ectopically expressing the $\exp R$ gene from the $\mathrm{pWB} \operatorname{expR}$ plasmid, called hereafter $\exp R^{+}$) [15], or even to the strain with a naturally occurring mutation in the AHL receptor-coding gene $\exp R$ (S. meliloti Rm2011 expR) [12] producing reduced amount of oxo-C14-HSL when compared to the S. meliloti $\exp R^{+}$strain (Figure 2B).

Figure 2. S. meliloti Rm2011 (expR) strain has lower, and S. meliloti Rm2011 (attM ${ }^{+}$) abolished AHL production in comparison to S. meliloti Rm2011 (expR $\left.{ }^{+}\right)$. All strains of $S$. meliloti were tested for the AHL production using the biosensor, $g f p$-expressing strain P. putida KS35 (A) AHL produced by $S$. meliloti strains were extracted with $\mathrm{CHCl}_{3}$ and applied onto a lawn of $P$. putida KS35 bacteria. The GFP signal was observed $2 \mathrm{~h}$ thereafter. S. meliloti attM $M^{+}$constitutively expresses the attM gene from A. tumefaciens coding for a lactonase. $S$. meliloti $\exp R$ has a natural mutation in the AHL receptor gene $\operatorname{expR}$. S. meliloti $\exp R^{+}$ectopically expresses $\operatorname{expR}$ gene; (B) Growth curves of S. meliloti strains with different AHL production.

A

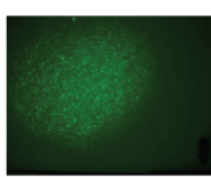

S. meliloti $\operatorname{expR}$

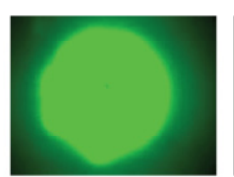

S. meliloti $\exp R^{+}$

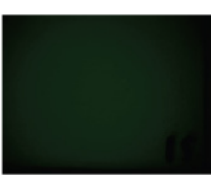

S. meliloti attM $^{+}$

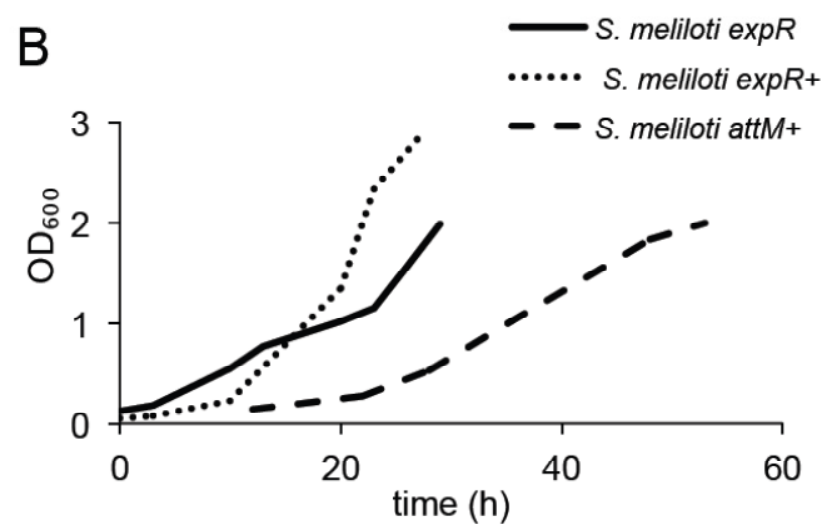

P. putida gfp-expressing biosensor 


\subsection{Plants Impact on AHL Concentration}

Next, we addressed the question whether plants influence the concentration of AHLs in their surroundings. To this end, we monitored the concentration of oxo-C14-HSL produced by $S$. meliloti $\mathrm{Rm} 2011 \operatorname{expR} R^{+}$in bacterial culture in the presence or absence of $A$. thaliana. AHLs were monitored in filtrates from bacterial cultures grown at $22{ }^{\circ} \mathrm{C}$ until the $\mathrm{OD}_{600 \mathrm{~nm}}=2.3$. After extraction with $\mathrm{CHCl}_{3}$, the solvent was evaporated and the residue resuspended in $\mathrm{Me}_{2} \mathrm{CO}$. AHLs were applied onto a lawn of the bacterial $g f p$-expressing biosensor strain P. putida KS35. The GFP signal was analyzed $2 \mathrm{~h}$ thereafter and quantified using the segmentation algorithm (Figure 3A,B). The concentration of oxo-C14-HSL reached a maximum at the late exponential phase $\left(\mathrm{OD}_{600 \mathrm{~nm}}=1.5\right)$ and decreased during the stationary phase (at $\mathrm{OD}_{600 \mathrm{~nm}}>2.3$ ) (Figure 3A,B). However, if Arabidopsis plants were co-cultivated together with the $S$. meliloti bacteria, the AHL concentration peak was missing and the amount of AHLs was reduced (Figure 3A,B). This result suggests that plants have a negative impact on either the AHL production, on their stability or availability. Whether the effect originates from Arabidopsis-produced lactonases, inhibition of AHL production or adhesion of AHLs to plant cell walls is not yet known. The answer to this question requires further tests.

Figure 3. The presence of plants has a negative impact on the accumulation of AHLs produced by $S$. meliloti. (A) Accumulation of AHL produced by $S$. meliloti expR $R^{+}$in culture with and without Arabidopsis plants was monitored using the bacterial biosensor strain P. putida KS35; (B) Quantification of the AHL production using a segmentation algorithm. Five photographs were taken per time point (see (A)) and tested for the ratio of GFP-positive pixels. The experiment was repeated five times. Error bars represent the S.D. between biological replicates. * represents $p>0.05$ in Student's $t$-test.

A

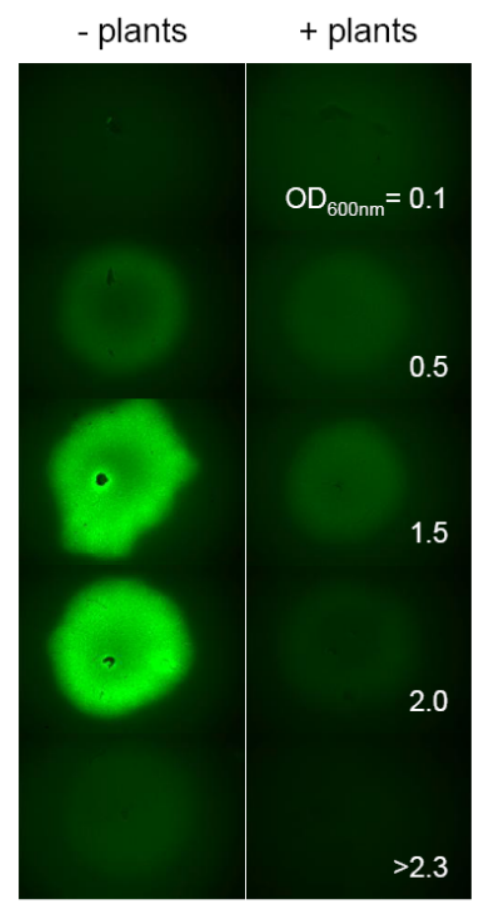

B

$\square-$ plants
+ plants

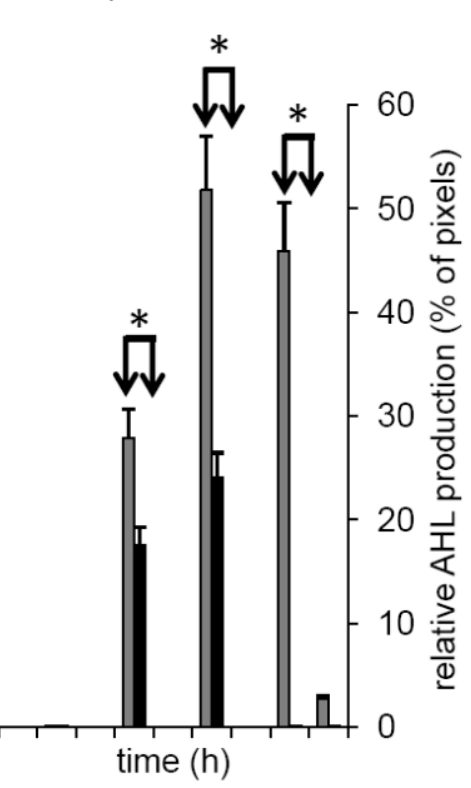




\subsection{Oxo-C14-HSL Produced by S. meliloti Enhances Resistance of Arabidopsis Plants}

Long-chain AHLs (e.g., oxo-C14-HSL) were shown to increase the resistance of barley and Arabidopsis plants towards hemibiotrophic pathogens; however, they show no effect on plant development $[8,10]$. To further substantiate our observation, we tested the impact of inoculation with S. meliloti strains producing oxo-C14-HSL on Arabidopsis resistance towards Pseudomonas syringae pv tomato (Pst) bacteria. We chose Arabidopsis rather than the native host of $S$. meliloti, Medicago tuncatula, in order to avoid effects related to nodulation and $\mathrm{N}_{2}$-fixation. The rhizosphere of soil-grown Arabidopsis plants was inoculated with different S. meliloti strains during a three-week period prior to the challenge. Pst bacteria were infiltrated into leaves and colony forming units (cfu) number was monitored one and $48 \mathrm{~h}$ after infiltration. In plants pre-treated with $S$. meliloti $\exp R^{+}$, the proliferation of Pst bacteria was significantly slower than in control plants (Figure 4A). Moreover, the resistance-inducing effect seems to be AHL-dependent since pre-treatment with strains producing less or no oxo-C14-HSL ( $S$. meliloti expR or S. meliloti att $M^{+}$, respectively) has a reduced or no effect on resistance (Figure 4A).

The expression profiles of two pathogenesis-related (PR) genes: PRI and Pdf1.2, were in agreement with those results. Challenge with flg22 induced the expression of $P R 124 \mathrm{~h}$ after treatment in all plants (Figure 4C). However, in plants pre-treated with extract originating from S. meliloti $\exp R^{+}$or $S$. meliloti $\exp R$, the induction of $P R 1$ was drastically increased (Figure 4C). Similarly, expression of $P d f 1.2$ was increased in plants pre-treated with extract from $S$. meliloti $\exp R^{+}$, though not in control or plants pre-treated with extract from $S$. meliloti strains producing less or no AHLs, S. meliloti expR or S. meliloti attM $M^{+}$(Figure 4D). Together with the induced resistance towards Pst, these results suggest that the pre-treatment with the S. meliloti strains producing oxo-C14-HSL (Figure 4E) prime Arabidopsis plants for better defense responses. In such primed state plants are able to respond in a faster and/or stronger way to a secondary challenge (e.g., pathogen infection).

Notably, this priming seems to be independent of the previously described priming mechanisms.

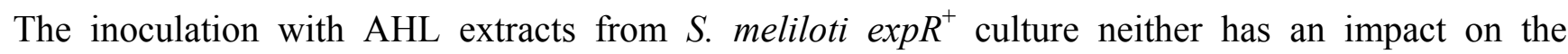
expression of the ISR associated $M Y C 2$ and $M y b 72$ transcriptions factors (Figure 5A,B), nor on their expression of those genes after a secondary challenge with flg22 (Figure 5C,D) if compared to control plants. Nevertheless, we observed an increase in the expression of both Myb72 and MYC2 after the challenge with flg22, in plants pretreated with extracts from either S. meliloti expR or S. meliloti attM ${ }^{+}$ (Figure 5C,D). These results suggest that the long-chain oxo-C14-HSL from $S$. meliloti interferes with the flg22-induced expression of both ISR associated genes, a phenomenon observed also for the treatment with pure oxo-C14-HSL [16]. The exact mechanism of this interaction is still not clear. 
Figure 4. The $S$. meliloti strains producing oxo-C14-HSL enhance resistance in Arabidopsis plants. The enhancement in resistance towards the pathogenic bacteria in Arabidopsis is dependent on AHL. (A) Proliferation of the plant-pathogenic P. syringae DC3000 (Pst) on plants pretreated with $\mathrm{MgCl}_{2}$ (control) or different S. meliloti strains, producing different quantities of oxo-C14-HSL. ${ }^{* * *} p>0.0005 ; * p>0.05$ in Student's $t$-test. cfu; colony forming unit (B) Symptoms caused by infiltration with Pst bacteria into Arabidopsis leaves; (C) and (D) Expression of the defense related Pathogenicity Related 1 $(P R 1)$ (C) and Pdf1.2 (D) genes. Expression was normalized to the expression of the At5g25760 gene (UBQ). Total RNA was extracted from 2-week-old Arabidopsis seedlings pre-treated for 3 days with AHL extract from different $S$. meliloti cultures and challenged with $100 \mathrm{nM}$ flg22 for hours as indicated. Graphs present a representative experiment from three independent replicates; (E) AHL production in S. meliloti strains used in the above experiments detected by the bacterial biosensor strain $P$. putida KS35.

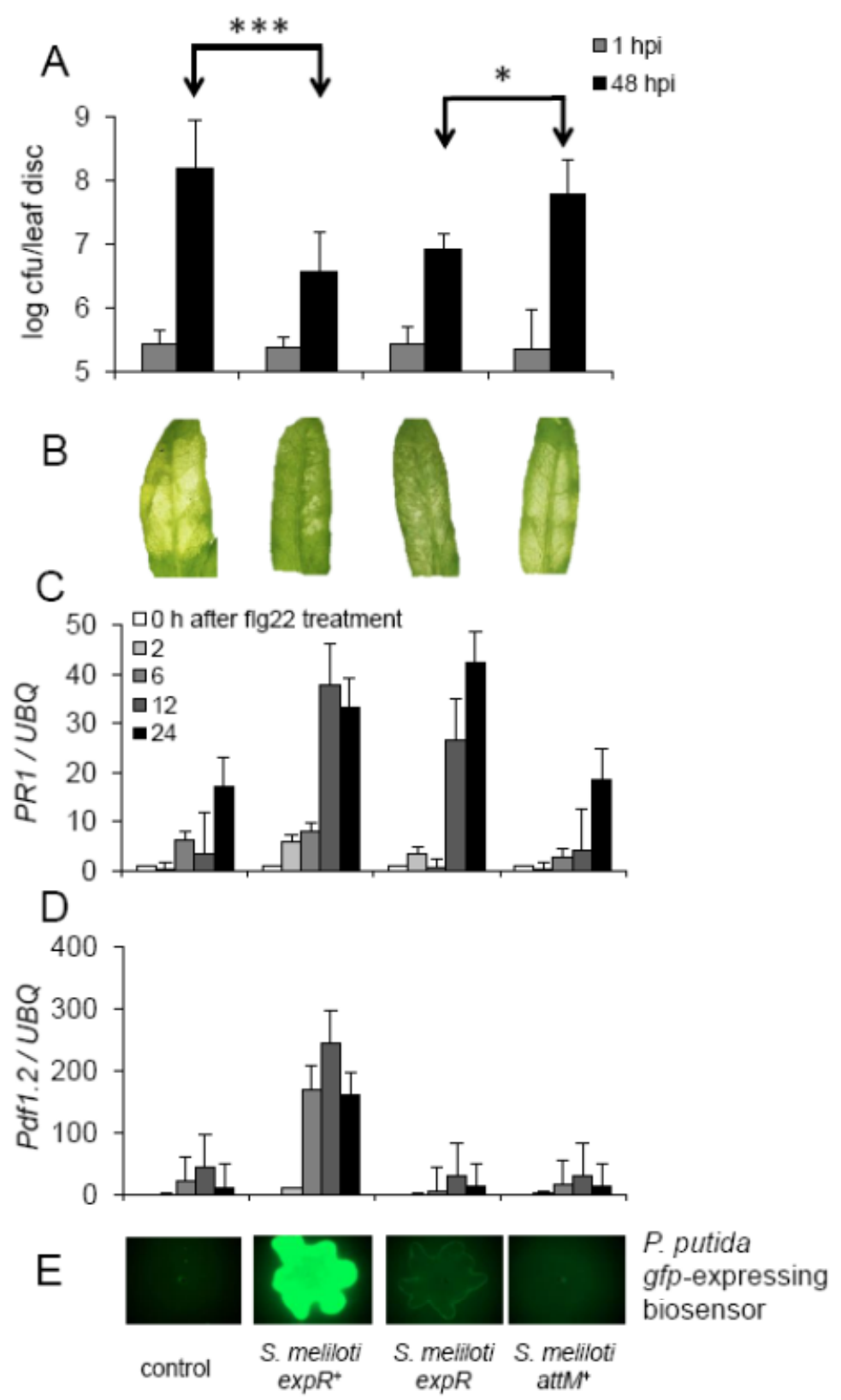


Figure 5. Expression of two Induced Systemic Resistance (ISR) related genes. Two-week-old Arabidopsis seedlings were pre-treated with AHL extract from different $S$. meliloti cultures, and challenged with $100 \mathrm{nM}$ flg22. (A) and (B) Expression levels of $M Y C 2$ (A) and Myb72 (B) after 3 days of pre-treatment; (C) and (D) Expression levels after the secondary challenge with $100 \mathrm{nM}$ flg22. All values were normalized to the expression of $U B Q$ gene. Graphs present a representative experiment from three independent replicates.

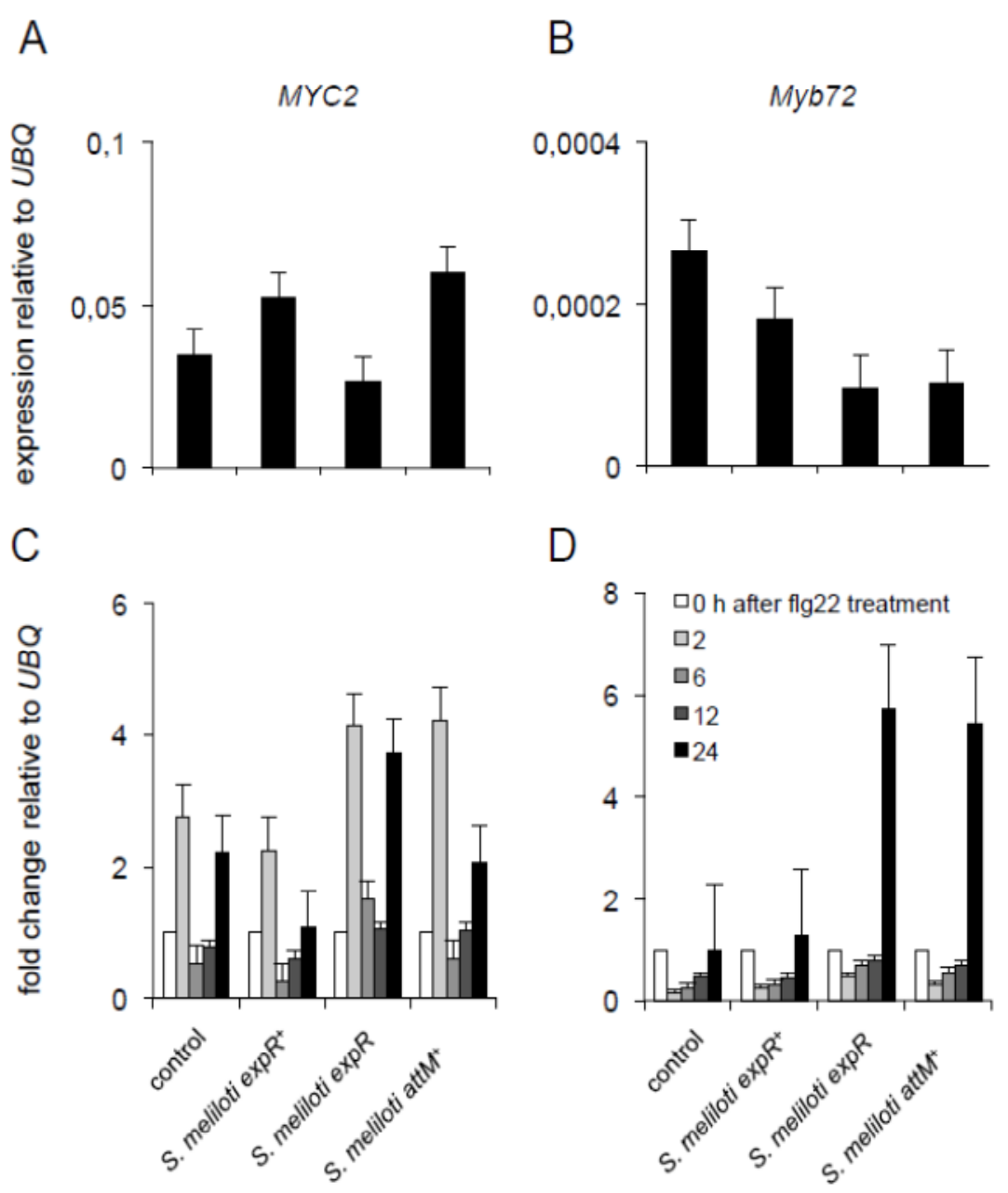

Next, we examined whether the inoculation with $S$. meliloli has an impact on Arabidopsis growth. To this end, we transferred one-week-old seedlings on vertical plates and inoculated them with S. meliloti $\exp R^{+}, S$. meliloti expR, and $S$. meliloti att $M^{+}$. The root tip positions were measured during 9 days after inoculation and pants weight was measured after 15 days. We observed no differences between the control plants and any of the treatments (Figure 6), suggesting that neither the $S$. meliloti nor its long-chain AHL has an impact on Arabidopsis growth. 
Figure 6. $S$. meliloti strains producing oxo-C14-HSL has no impact on the growth of Arabidopsis plants. (A) Root length of Arabidopsis plants co-inoculated with S. meliloti strains for 9 days; (B) Fresh weight of shoots of plants inoculated with $S$. meliloti strains during 15 days; (C) AHL production by $S$. meliloti strains used in the above experiments on Arabidopsis roots detected by the biosensor bacteria $P$. putida KS35. Student's $t$-test revealed no differences between control and the different treatments, at $p \geq 0.05$ in values presented in (A) and (B).

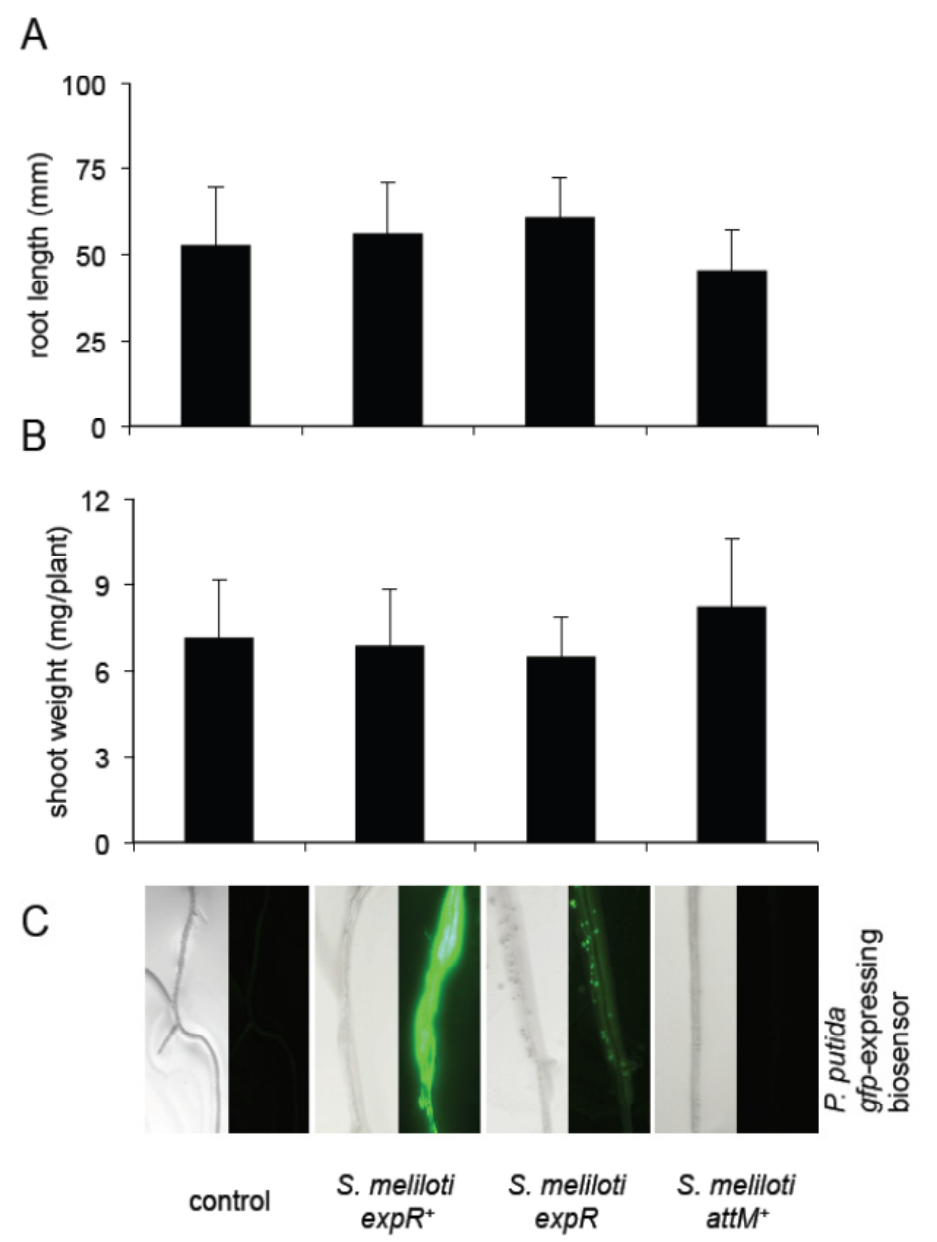

\subsection{Oxo-C8-HSL Produced by R. etli Has only Moderate Impact on Arabidopsis Growth}

Previous reports on short-chain (C6-HSL and oxo-C8-HSL) AHLs influence on plants suggested that those molecules possess a growth-promoting effect $[6,8,17]$. Therefore, in the second part of this study, we addressed the question whether the presence of rhizobia producing short-chain AHLs would have a positive impact on plants. $R$. etli was previously shown to produce oxo-C8-HSL and was chosen in this study [18]. In the first step, we verified the nature of the AHLs produced by $R$. etli 11541 under our conditions. A peak overlapping with oxo-C8-HSL was identified by LC-MS. The presence of oxo-C8-HSL was subsequently confirmed by MS/MS (Figure 7). This result is in line with the previous reports on AHL production by $R$. etli. The identity of oxo-C8-HSL was unambiguously confirmed by the presence of the following adduct ions $m / z: 241.9\left([\mathrm{M}+\mathrm{H}]^{+}\right), 264.0\left([\mathrm{M}+\mathrm{Na}]^{+}\right)$, $483.0\left([2 \mathrm{M}+\mathrm{H}]^{+}\right)$and $505.1\left([2 \mathrm{M}+\mathrm{Na}]^{+}\right)$. The pseudomolecular ion $241.9\left([\mathrm{M}+\mathrm{H}]^{+}\right)$was subsequently selected as a precursor ion for unambiguous HR-MS/MS identification $\mathrm{m} / \mathrm{z}$ : 242.1326 
$\left(\mathrm{C}_{12} \mathrm{H}_{19} \mathrm{NO}_{4},[\mathrm{M}+\mathrm{H}]^{+}\right), 141.0898\left(\mathrm{C}_{8} \mathrm{H}_{13} \mathrm{O}_{2}{ }^{+}\right), 102.0532\left(\mathrm{C}_{4} \mathrm{H}_{8} \mathrm{NO}_{2}{ }^{+}\right), 74.0587\left(\mathrm{C}_{3} \mathrm{H}_{8} \mathrm{NO}^{+}\right), 71.0829$ $\left(\mathrm{C}_{5} \mathrm{H}_{11}{ }^{+}\right)$and $56.0491\left(\mathrm{C}_{3} \mathrm{H}_{6} \mathrm{~N}^{+}\right)$. All diagnostic adduct and fragment ions listed above were confirmed by analyzing the reference standard. In addition, in analogy to $S$. meliloti, we constructed two AHL-negative strains in which the accumulation of oxo-C8-HSL was abolished due to the expression of the att $M$ lactonase gene of $A$. tumefaciens using two different pBBR-variants conferring kanamycin and gentamicin resistance, respectively (Figure S2).

Figure 7. Verification of AHLs production in Rhizobium etli 11541. HPLC samples were screened in the positive ion mode, and a scan from $\mathrm{m} / \mathrm{z} 50$ to 500 was performed. (A) The MS of standard oxo-C8-HSL standard $\left(\mathrm{m} / z=242.1[\mathrm{M}+\mathrm{H}]^{+}\right)$shows a pattern similar to AHLs extracted from the $R$. etli culture; (B) Further analysis by MS/MS confirmed the identity of AHL extracted from the $R$. etli culture with the standard oxo-C8-HSL. The diagnostic fragment ions $\mathrm{m} / \mathrm{z} 102.0532$ and 102.0537 are indicative for the presence of the lactone ring. The highlighted $\mathrm{m} / \mathrm{z}$ values represent diagnostic fragment ions. Diamonds mark the pseudomolecular ions used for MS/MS analysis.

\section{A}

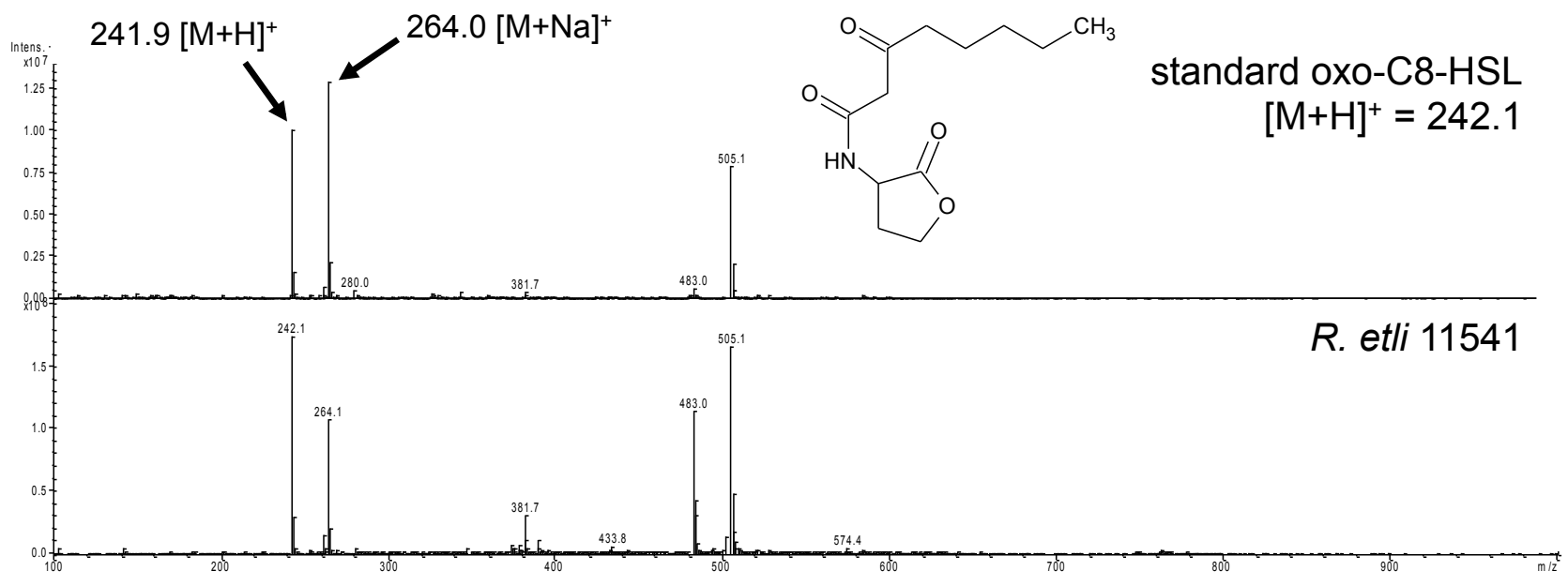

B

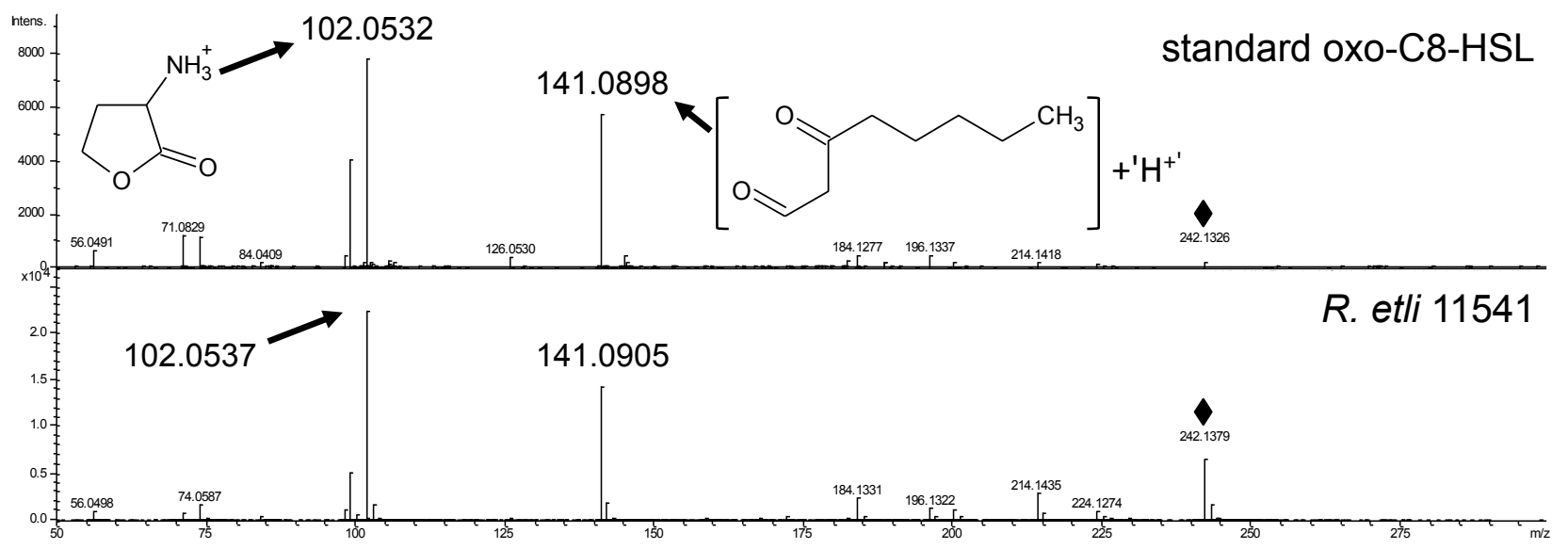


In the next step, we assessed whether the oxo-C8-HSL produced by $R$. etli (Figure 7) influences the growth of Arabidopsis. In line with the tests with $S$. meliloti, we chose Arabidopsis rather than the native host (Phaseolus vulgaris) in order to avoid the nodulation and $\mathrm{N}_{2}$-fixation related phenotype, which could mask the AHL effect. Besides, R. etli had been already reported to be a plant growth promoting rhizobacterium (PGPR) on non-legume plants [19]. To verify the effect of $R$. etli-originated oxo-C8-HSL, one-week-old Arabidopsis seedlings were transferred to a vertical growth system (square Petri dishes with half MS medium and no sucrose supply) and inoculated with $R$. etli wild type (wt), R. etli attM $M^{+}\left(\mathrm{Km}^{R}\right)$, and $R$. etli $\mathrm{attM}^{+}\left(\mathrm{Gm}^{R}\right)\left(\mathrm{OD}_{600 \mathrm{~nm}}<0.1\right), 10 \mathrm{mM} \mathrm{MgCl}$ solution was used as a control. The root tip position was measured every 3rd day. After 9 days we observed no differences between plants inoculated with $R$. etli wt and those treated with $\mathrm{MgCl}_{2}$ solution (Figure $8 \mathrm{~A}$ ). However, plants inoculated with both lactonase-expressing strains: $R$. etli attM $M^{+}\left(\mathrm{Km}^{R}\right)$ and $R$. etli attM $\left(\mathrm{Gm}^{R}\right)$ showed significantly shorter roots (Figure $8 \mathrm{~A}$ ). Likewise, the shoot weight measured 15 days after inoculation was similar between controls and $R$. etli wt treated plants (Figure 8B). In contrast, plants inoculated with $R$. etli att $M^{+}\left(\mathrm{Gm}^{R}\right)$ had significantly lighter rosettes (Figure 8B). We hypothesize that the oxo-C8-HSL produced by the wild type $R$. etli strain (Figure 8C) balances the otherwise negative impact of $R$. etli colonization on Arabidopsis growth.

Figure 8. $R$. etli producing oxo-C8-HSL has only moderate impact on Arabidopsis growth. (A) Root length of Arabidopsis plants co-inoculated with the indicated $R$. etli strains for 9 days; (B) Fresh weight of shoots of plants inoculated with $R$. etli strains during 15 days; (C) AHL production by $R$. etli strains used in the above experiments on Arabidopsis roots detected by the biomarker bacteria $E$. coli. Letters indicate statistical differences in Student's $t$-test at $p \geq 0.005$ (A) or $p \geq 0.05$ (B), dai; day after inoculation.

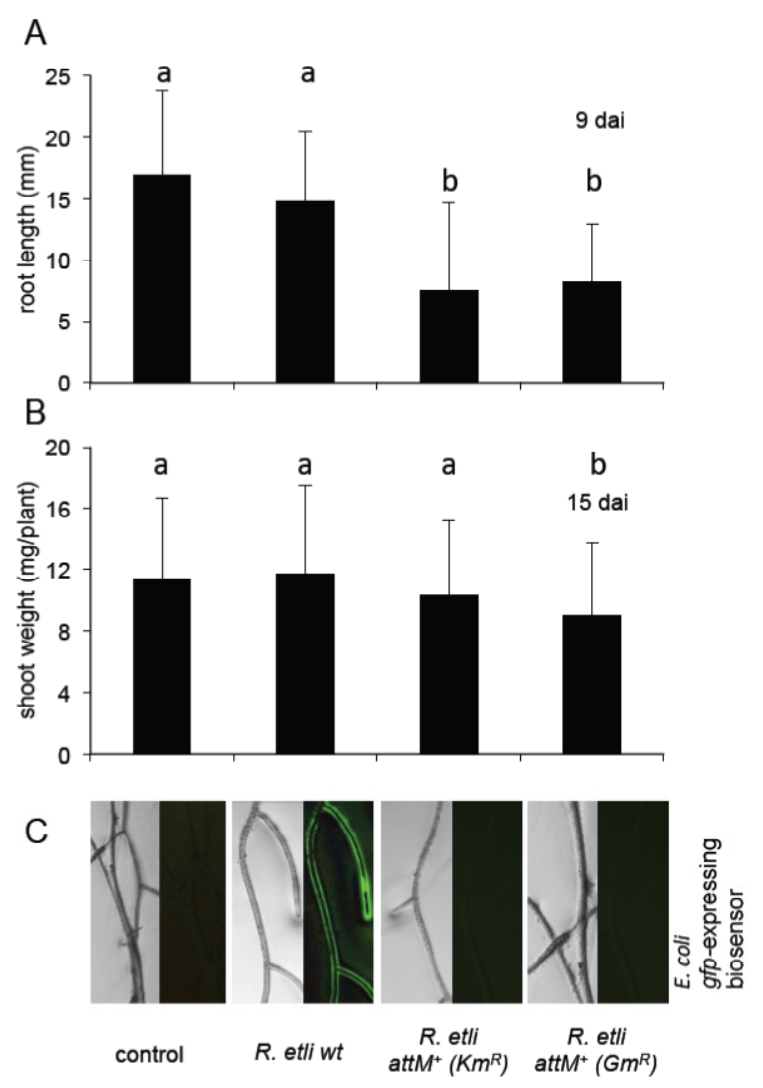


A possible explanation why Arabidopsis reacts to $R$. etli colonization with a growth inhibition could be an induced defense mechanism. To prove this assumption we tested the resistance towards the hemi-biotrophic Pst bacteria. Two different infection methods with Pst were chosen: infiltration and spray-inoculation. Soil-grown Arabidopsis Col-0 wild type plants were watered with $R$. etli wt, R. etli attM $M^{+}\left(\mathrm{Km}^{R}\right)$ and $R$. etli attM $M^{+}\left(G m^{R}\right)(\mathrm{OD}<0.1)$ suspension during three weeks prior challenge with $P s t$ bacteria. Cfu of Pst were analyzed one and $48 \mathrm{~h}$ after infiltration in leaf discs. We observed no differences in the resistance or susceptibility towards Pst (Figure S3A). Similarly, the spray-inoculation of hydroponically grown Arabidopsis with Pst revealed no difference in the resistance towards this pathogen (Figure S3B), suggesting that $R$. etli has no influence on the resistance towards $P$. syringae in Arabidopsis plants.

\section{Discussion}

In this report, we investigated the impact of bacteria-originated AHLs at defense responses and growth of $A$. thaliana. Both bacterial species used in this study are well-studied rhizobia entering close symbiosis with legumes. On the one hand, we chose $S$. meliloti, which produces several AHLs, including oxo-C14-HSL [11,20]. As recently shown, long-chain AHLs induce resistance in Arabidopsis and barley plants $[8,10]$. Here, we show the positive effect of a strain producing high amounts of oxo-C14-HSL ( $S$. meliloti $\exp R^{+}$) on plant resistance. As expected, this bacterial strain has no influence on plant growth. We propose an AHL-induced priming as the mechanism of increased resistance. Interestingly the AHL-induced priming seems to be different from the priming induced by other PGPR, as shown by the expression of $M Y C 2$ and $M y b 72$ transcription factors [21,22]. Notably, the AHL synthase $(\sin I)$ and AHL receptor $(\exp R)$ expressed in free-living rhizobia are repressed in bacteria living in a symbiosis with the native host $M$. truncatula [23]. We therefore hypothesize, that in case of nodule-living bacteria the AHL-priming effect is missing. On the other hand, we confirmed our previous findings that short-chain AHLs, like oxo-C8-HSL from $R$. etli, have no resistance-inducing activity. Unfortunately, the expected positive effect of bacterial oxo-C8-HSL on plant growth is only very moderate; indicating that the interaction between plant and rhizobia depends on more signals than AHLs. Taken together this study provides new arguments supporting the observation that plants respond to bacterial AHLs and that this response depends on the length of the AHLs.

\subsection{AHL Production in S. meliloti and R. etli}

The impact of bacterial AHLs on plants was postulated in several independent studies [3,6,10,17,24]. However, only recent results indicated that different molecules might have different influence on plant hosts [8]. Others and our studies suggested that the short-chain AHLs increase the growth of Arabidopsis plants [6-8,17], whereas the long-chain AHLs reinforce the plant resistance against biotrophic and hemibiotrophic pathogens [10]. Therefore, the fact that the bacteria chosen in this study produce either long or short-chain AHLs allowed to verify our previous hypothesis on AHLs bi-functionality in respect to plant reactions [25]. Very remarkable was the specificity in the AHL type. Both bacterial species, $S$. meliloti and $R$. etli, were already examined in this regard, the AHLs identified in this report were previously reported to be within the palette of homoserine lactones produced by the respective bacterial species $[11,18,20]$. 


\subsection{Systemic Induction of Arabidopsis Resistance by S. meliloti Treatment}

Previous studies have shown that S. meliloti produces different long-chain AHLs including 3-oxo-C14-, C16-, 3-oxo-C16-, C16:1-, and 3-oxo-C16:1-HSL [26,27]. In this study, we confirmed the presence of oxo-C14-HSL in S. meliloti culture. Because of the previously observed, positive impact of oxo-C14-HSL treatment on Arabidopsis and barley resistance [10], we expected that $S$. meliloti strains producing oxo-C14-HSL will have positive effect on Arabidopsis resistance. Indeed, co-cultivation of Arabidopsis plants with the $S$. meliloti $\exp R^{+}$strain, which produces high amounts of oxo-C14-HSL, significantly enhances the resistance towards the pathogenic Pst bacteria. In addition, also the

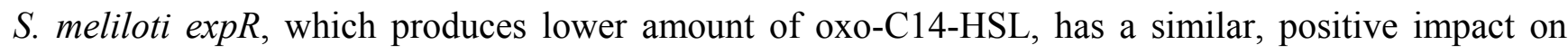
plant resistance. In the same context, plants pretreated with the lactonase-expressing S. meliloti attM ${ }^{+}$ strain showed increased Pst proliferation when compared to plants pretreated with the oxo-C14-HSL- producing S. meliloti strains. These results indicate an involvement of the AHL in the resistance conferred by S. meliloti. Similar results have been obtained by Schuhegger et al. [3], these authors reported the induction of resistance against the fungal leaf pathogen Alternaria alternata, in tomato plants pretreated with Serratia liquefaciens MG1. Furthermore, they indicated that the AHL-negative mutant of $S$. liquefaciens MG44 was less effective in induction of resistance against A. alternata [3]. Intriguing is the fact that although the major AHL produced by S. liquefaciens is the short-chain C6-HSL [28], it appears to confer resistance to a necrotrophic pathogen. Unfortunately, we were not able to verify this effect with pure AHL or the oxo-C8-HSL-producing $R$. etli strain (data not shown), leaving the question on the impact of short-chain AHLs on resistance towards necrotrophic pathogens open. Interesting to note are the different capabilities of moving throughout a plant of short and long-chain AHLs, while the short-chain C6-HSL was found in shoot of Arabidopsis when applied to root, the long-chain AHL oxo-C14-HSL was not [10]. In addition, also the substitutions in the lipid chain play a role in induced resistance [10], indicating that besides the specific response to a given AHL, plants possess also systemic signal, which regulates the AHL-priming.

\subsection{Growth Inducing Capacities}

Short-chain AHL was shown to promote growth in Arabidopsis plants [6-8,17]. Depending on the exact length, the possible effect may include also alteration in root hair morphology and thickening of roots [17]. The short-chain oxo-C8-HSL produced by $R$. etli (this work and [18]) encouraged us to test whether the inoculation with AHL-producing bacteria has effect similar to the pure molecule. As concluded above, despite the fact that it has been already reported to be a plant growth promoting rhizobacterium (PGPR) on the non-legume tomato and pepper plants [19], $R$. etli has a negative effect on Arabidopsis growth. However, this negative impact is possibly contra-balanced with the positive effect of oxo-C8-HSL produced by this bacterium. Such compensation phenomenon could be the explanation for the reduced growth of plants pretreated with the lactonase-expressing strains (R. etli attM $M^{+}, \mathrm{Km}^{R}$ and $G m^{R}$ ), in comparison to growth at nearly control level of plants pretreated with the wild type $R$. etli producing short-chain AHL. These results appear to be in line with reports that only a few rhizobacteria are known to be naturally associated with Arabidopsis roots, and only few showed positive growth effects [29]. As expected, treatment with S. meliloti has no influence on the 
growth of Arabidopsis. Neither the $S$. meliloti $\exp R^{+}$, producing high levels of AHLs, nor the AHL-negative (S. meliloti att $M^{+}$) strain influenced the growth rate of Arabidopsis.

\subsection{Impact of Plant on AHL Production}

Several previous reports demonstrated the effect of AHLs on biofilm formation in bacterial communities. Recently, a model assuming the role of autoinducers in promotion of highly adaptable, spatial heterogeneity in populations was suggested [30]. Notably, the presence of plants diminished the concentration of AHL in bacterial culture, without major effect on the bacterial proliferation (Figure S4). While some explanations are possible: adhesion to root surface or a residual lactonase activity of the five phosphogluconolactonases encoded on Arabidopsis genome, the most probable is however a quorum quenching (QQ) activity of root exudates. Such activity was already reported for several higher plants and algae [31-36]. Between many unidentified QS-mimicking and QQ molecules in root exudates, halogenated furanones, $L$-canavanine and flavan-3-ol catechin have been identified as plant-originated inhibitors of QS [34-36]. Especially the halogenated furanones from the red alga Delisea pulchra act directly at the AHL reception site by facilitating the degradation of the AHL-LuxR complex and therefore inhibiting of QS-depending processes [34]. Whether higher plants secret similar compounds was not yet reported. A screening for QS active substances in the native host of S. meliloti, Medicago truncatula, revealed 15 to 20 substances, which either activate or inhibit QS [32]. A similar study identified QS-interfering activities in exudates from rice and bean plants [33]; however, the nature of those molecules remains undetermined. Whether Arabidopsis exudates compound(s) with similar activity is probable, though not yet proven. Very intriguing is also the possibility that plant-originated molecules influence not only the perception or stability of QS molecules, but also the synthesis.

Taken together this report demonstrates the dependence of the plant reaction to rhizobia on the type of AHL, produced by those bacteria. The AHL-priming effect detected in plants pre-treated with oxo-C14-HSL producing S. meliloti strains shows that the complex interaction between bacteria and plants goes further than the perception of MAMPs and effectors or even NOD factors. Additional experiments shall clarify the mechanisms of AHL-priming and its potential in agriculture.

\section{Experimental Section}

\subsection{Plant}

Wild type Arabidopsis thaliana Col-0 (ecotype Columbia) was obtained from The Nottingham Arabidopsis Stock Center (NASC), NASC ID: N60000. Plants were either grown on soil under short-day conditions $\left(8 / 16 \mathrm{~h}\right.$ light regime, at $\left.21{ }^{\circ} \mathrm{C}\right)$ or in sterile condition on half MS medium supplemented with $0.8 \%$ agar at $21{ }^{\circ} \mathrm{C}$. For systemic approach, plants were grown in sterile hydroponics culture using half MS medium without sucrose, assuring the separation between roots and shoot parts.

\subsection{Bacterial Strains and Growth Conditions}

R. etli 11541 wild type was obtained from DSMZ (German Collection of Microorganisms and Cell Cultures) in Braunschweig, Germany. S. meliloti Rm2011, an $\exp R$ mutant (expR), was obtained from 
A. Becker. S. meliloti Rm2011 expR $R^{+}$containing the $\mathrm{pWB} \operatorname{expR}$ plasmid was obtained from M. McIntosh. All used $S$. meliloti strains are resistant to streptomycin $(250 \mu \mathrm{g} / \mu \mathrm{L})$.

R. etli (pBBR2-attM), R. etli (pBBR5-attM) and S. meliloti (pBBR2-attM) carrying the lactonase gene attM from Agrobacterium tumefaciens were obtained by conjugation with Escherichia coli S17-1 carrying attM on pBBR1MCS-2 (conferring kanamycin resistance, $\mathrm{Km}^{R}$ ) or pBBR1MCS-5 (conferring gentamicin resistance, $G m^{R}$ ) plasmids [37].

Rhizobia were grown in $5 \mathrm{~mL}$ culture in a $50 \mathrm{~mL}$ Erlenmeyer flask under $100 \mathrm{rpm}$ constant agitation at $21^{\circ} \mathrm{C}$ in TY medium [13]. The OD was measured every $4 \mathrm{~h}$ using $50 \mu \mathrm{L}$, we performed dilutions for ODs above 0.8 .

E. coli MT102 (pJBA89) resistant to ampicillin $100 \mu \mathrm{g} / \mathrm{mL}$ [38], and P. putida KS35, resistant to gentamicin $(20 \mu \mathrm{g} / \mathrm{mL})$ and kanamycin $(50 \mu \mathrm{g} / \mathrm{mL})$ [39] were used for AHLs detection.

\subsection{AHL Detection and Quantification}

\subsubsection{Chemicals}

All solvents used for LC/MS analyses, acetonitrile (MeCN, 99.9\%), and formic acid (FA, 98\%), were of LC/MS grade from Sigma-Aldrich (Steinheim, Germany). Water was purified by a Merck-Millipore Milli-Q Synthesis A10 system (Merck-Millipore, Schwalbach/Ts., Germany).

\subsubsection{HPLC/MS-MS}

In order to identify the type of AHLs produced by $R$. etli and $S$. meliloti, bacteria were grown on TY media $\left(\mathrm{OD}_{600 \mathrm{~nm}} \approx 0.8\right)$ with the respective antibiotics in a large scale $(80 \mathrm{~mL})$. The cultures were centrifuged to remove the bacterial cells, and $5 \mathrm{~mL} \mathrm{CHCl}_{3}$ was added for extraction of AHLs. AHL extract were evaporated and redissolved in $80 \% \mathrm{MeCN}$, vortexed, placed for $15 \mathrm{~min}$ in an ultrasonic bath (Sonorex, Bandelin, Berlin, Germany) and centrifuged for $15 \mathrm{~min}$ at 21,000× $g$ (Mikro 220R, Hettich, Tuttlingen, Germany). For LC/MS analysis and fractionation, two mass spectrometers, both from Bruker Daltonics (Bremen, Germany), were used. Both instruments were controlled by the HyStar software (version 3.2, SR 2, Bruker Daltonics, Bremen, Germany, 2012). The instruments were equipped with an orthogonal ESI source.

For separation and fractionation of different AHL-types, an amaZon ETD Ion-Trap MS, coupled to a Dionex UltiMate 3000 HPLC (Dionex, Idstein, Germany), has been used. Samples were separated on an Acclaim $120 \mathrm{C} 18,3 \mu \mathrm{m}, 120 \AA, 4.6 \times 150 \mathrm{~mm}$ column (Dionex, Idstein, Germany) at a flow rate of $1 \mathrm{~mL} / \mathrm{min}$ and at $35{ }^{\circ} \mathrm{C}$. Eluent A consisted of $\mathrm{H}_{2} \mathrm{O}+0.1 \% \mathrm{FA}$, eluent B of $80 \% \mathrm{MeCN}+0.1 \%$ FA. Samples of $150 \mu \mathrm{L}$ were injected. A linear gradient of $5 \%-100 \%$ B in 40 min was used. The source parameters were adjusted as follows: capillary voltage $4500 \mathrm{~V}$; end plate offset $500 \mathrm{~V}$; nebulizer 1 bar, dry gas $8 \mathrm{~L} / \mathrm{min}$ with a dry temperature of $200{ }^{\circ} \mathrm{C}$. The mass accuracy of the low-resolution system is $\pm 0.2 \mathrm{Da}$. Before injection into the Ion-Trap MS, the flow was split: one third was used for monitoring of the different AHLs in the positive ion mode using a full scan from $\mathrm{m} / z 100$ to 2000. Two thirds were used to fractionate the samples. Over a time period of $80 \mathrm{~min}$, fractions were collected every $30 \mathrm{~s}$. These fractions were evaporated using ultra-speed vacuum centrifugation and 
redissolved in $80 \% \mathrm{Me}_{2} \mathrm{CO}$ (acetone) in order to be suitable for the GFP biosensor bacteria assay described below. Only bioactive fractions were used for subsequent identification.

A high-resolution microTOF-Q II mass spectrometer was used for identification of bioactive secondary metabolites. For direct infusion experiments, samples were applied via syringe pump at a flow rate of $180 \mu \mathrm{L} / \mathrm{h}$. The source parameters were adjusted as follows: capillary $4500 \mathrm{~V}$, end plate offset $500 \mathrm{~V}$, nebulizer $0.4 \mathrm{Bar}$, dry gas $4 \mathrm{~L} / \mathrm{min}$, dry temprature $180^{\circ} \mathrm{C}$. The ion optics were adjusted as follows: funnel $1 \mathrm{RF} 200 \mathrm{Vpp}$, funnel $2 \mathrm{RF} 200 \mathrm{Vpp}$, ISCID energy $0 \mathrm{eV}$, hexapole RF $100 \mathrm{Vpp}$, ion energy $3 \mathrm{eV}$, collision RF $180 \mathrm{Vpp}$, collision energy $8 \mathrm{eV}$, transfer time $80 \mu \mathrm{s}$, and pre puls storage $7 \mu \mathrm{s}$. The mass accuracy of the high-resolution system is $\pm 10 \mathrm{mDa}$. Positive-mode MS/MS experiments were performed in the $\mathrm{m} / \mathrm{z}$ range from 50 to 500. Precursor ions were fragmented at collision energy of 20-30 eV. Data interpretation was performed using the DataAnalysis software (version 4.0 SP 5, Bruker Daltonic, Bremen, Germany, 2012). Standards of C6-, oxo-C8-, oxo-C10-, oxo-C12-, and oxo-C14-HSLs from Sigma-Aldrich were used as reference standards in both systems.

\subsubsection{AHL Detection Using Biosensor Bacteria}

AHLs were extracted either from the liquid medium where plants were growing (as described in plant growth conditions) or from bacterial culture. All extractions were performed by adding $\mathrm{CHCl}_{3}$, centrifugation, and discarding the aqueous phase. $\mathrm{CHCl}_{3}$ was evaporated using ultra-speed vacuum centrifugation and the residue was redissolved in $\mathrm{Me}_{2} \mathrm{CO}$. In order to detect AHLs production, two reporter bacteria were used: Escherichia coli MT102 (pJBA89), which is able to detect all side chain lengths of AHLs, and P. putida KS35 that can perceive only long-chain AHLs. These $g f p$-expressing biosensor bacteria were grown on LB medium with the corresponding antibiotics over night at $21{ }^{\circ} \mathrm{C}$ and plated on LB agar plates for one more night. Five microliters of extracted and redissolved AHLs were dropped on the plates containing a lawn of the reporter bacteria and detection of GFP expression was done after $2 \mathrm{~h}$ by fluorescence binocular microscope.

\subsection{Segmentation Algorithm}

In order to objectively analyze the fluorescence response of the reporter bacteria a full automatic algorithm has been developed which returns the relative AHL production as a percentage of all pixels in the microscopic images. The key idea is to segment the image into two disjoint region sets. The first one will represent the fluorescing bacteria (foreground) and the second one the non-fluorescing parts (background) in the image. Given this segmentation, the quotient between the size of the foreground region set and the whole image size is the relative AHL production. The segmentation algorithm is an adaptation of the work presented in [40]. Here, an energy functional is minimized, whose minimum is a binary image representing the segmentation result. As input for this process, a user defines on an arbitrary image the foreground and background region. The algorithm trains from this a model how pixels and its neighbors appear for a given region. This model was used to evaluate all microscope images. 


\subsection{Growth Promotion Assay}

One-week-old Arabidopsis seedlings were transferred into half MS square plates without sucrose. The root tip position was marked and $10 \mu \mathrm{L}$ of bacterial cultures $\left(\mathrm{OD}_{600 \mathrm{~nm}}<0.1\right)$ were dropped on the root tip. Root length was measured manually every 3rd day. The roots and leaves weight was measured at the end of the experiment. The bacterial treatments included the indicated bacterial strains as well as $\mathrm{MgCl}_{2}$ used as a control.

\subsection{Pathogenicity Assays}

Two methods for Pseudomonas syringae pv. tomato DC3000 (Pst) quantification were tested. Roots of 6-week-old Arabidopsis thaliana Col-0 grown in a sterile systemic system were pretreated with rhizobia bacteria. After 3 days, shoots were spray-inoculated with $P s t$ at $\mathrm{OD}_{600 \mathrm{~nm}}=0.1$ in $10 \mathrm{mM}$ $\mathrm{MgSO}_{4}, 0.02 \%$ Silwet77. Leaves were harvested after $1 \mathrm{~h}$ and $96 \mathrm{~h}$ post inoculation (hpi) and homogenized. A serial of dilutions with $\left(10 \mathrm{mM} \mathrm{MgSO}_{4}\right)$ from the homogenized plant materials was made and dropped on King's B medium. The colony forming units (cfu) of Pst were counted 2 days later in order to assess the proliferation of Pst. In the second approach Arabidopsis plants were grown on soil for four weeks as described under plant growth conditions. Plants have been pretreated 3 times with (1-4 mL) of bacterial cultures grown until exponential phase. Three days after the last treatment, leaves where infiltrated with Pst $(\mathrm{OD} 600 \mathrm{~nm}=0.01)$ diluted in $10 \mathrm{mM} \mathrm{MgSO}_{4}$. A biopsy punch was used to prepare leaf discs with $5 \mathrm{~mm}$ diameter at $1 \mathrm{hpi}$ and $48 \mathrm{hpi}$. The leaf discs have been homogenized, diluted and used as described in the first pathogenicity assay to count the cfu of Pst.

\subsection{Gene Expression Analysis}

We used quantitative RT-PCR for all gene expression analyses. Total RNA was extracted with $1 \mathrm{~mL}$ Trizol (PeqLab, Erlangen, Germany) according to manufactures' protocol. Two $\mu \mathrm{g}$ of total RNA was used for DNaseI digestion. cDNA synthesis was followed according to the qScript cDNA Synthesis Kit from Quanta BioScience Inc. (Gaithersburg, MD, USA). In order to check the efficiency of the reverse transcription, a semi-quantitative amplification of the actin2 transcript (cycles: 28, annealing temp. $55^{\circ} \mathrm{C}$ ) was performed. A positive genomic DNA control was included to verify the purity of cDNA samples from a genomic DNA. Fifty nanogram cDNA was used for quantitative RT-PCR (Applied Biosystems 7500 real-time PCR system, Foster City, CA, USA). Annealing temperature was set to $60{ }^{\circ} \mathrm{C}$ in every qRT-PCR and the cycle number was set to 42 . All expression values were normalized to the expression of $U B Q$ gene and to the 0 hours post infection (hpi) values. Quantitative RT-PCR was done using specific primers: $A t 5 g 25760$ (UBQ) fwd.: GCTTGGAGTCCTGCTTGGACG rev.: CGCAGTTAAGAGGACTGTCCGGC; $\quad$ PR1 fwd.: GGaGCGGtAGGCGTAGGTCCC, rev.: CCCACGAGGATCATAGTTGC; Pdf1.2 frw.: GTTTGCTTCCATCATCACC, rev.: GGGACGTAACAGATACACTTG; Myb72 frw.: TCATGATCTGCTTTTGTGCTTTG, rev.: ACGAGATCAAAAACGTGTGGAAC; MYC2 frw.: TCATGATCTGCTTTTGTGCTTTG, rev.: ACGAGATCAAAAACGTGTGGAAC. 


\section{Conclusions}

The interaction between root-associated bacteria and plants encompasses many levels. In addition to the well-studied symbiotic interaction between members from the Fabaceae family and rhizobia and the diverse pathogenic bacteria and their hosts, we present here the impact of bacterial quorum sensing molecules on plants resistance. In agreement with observations done on the impact of pure molecules from the acyl-homoserine lactones group on plants, we show here that also bacteria-originated molecules have similar qualities. The inoculation with long-chain AHL (oxo-C14-HSL) producing S. meliloti strains enhances the resistance of Arabidopsis plants, while inoculation with a strain unable to accumulate oxo-C14-HSL did not have this effect. Moreover, the resistance enhancement depends on the length of the acyl side chain because the inoculation with short-chain (oxo-C8-HSL) producing $R$. etli had no effect on the defense mechanism. These results indicate that plant perceive the AHL in diversified manner. In addition, the AHL-producing bacteria could hold a potential for future agricultural applications.

\section{Acknowledgments}

The authors would like to thank Prof. Anton Hartmann (Helmholtz Center Munich, Germany) for providing the biosensor bacterial strains used in this work. We are grateful to M. McIntosh and A. Becker (Centre of Synthetic Microbiology, Marburg, Germany) for proving the plasmid pWBexpR and the strain S. meliloti Rm2011. The work of KHK and AS was supported by the Bundesanstalt für Landwirtschaft und Ernährung (BLE) grant Nr. 2811NA033.

\section{Conflicts of Interest}

The authors declare no conflict of interest. 


\section{Supplementary Information}

Figure S1. Detection and quantification of standard AHLs using the bacterial biosensor strains P. putida KS35 and E. coli E. coli MT102 (pJBA89). (a) The GFP-based system using the $P$. putida KS35 and E. coli MT102 (pJBA89) strains. AHLs were purchased from Sigma-Aldrich, resuspended in $\mathrm{Me}_{2} \mathrm{CO}$ at concentrations as indicated and $5 \mu \mathrm{L}$ of the solution were applied onto bacterial lawns; (b) Quantification of the GFP photographs using the segmentation algorithm [40].

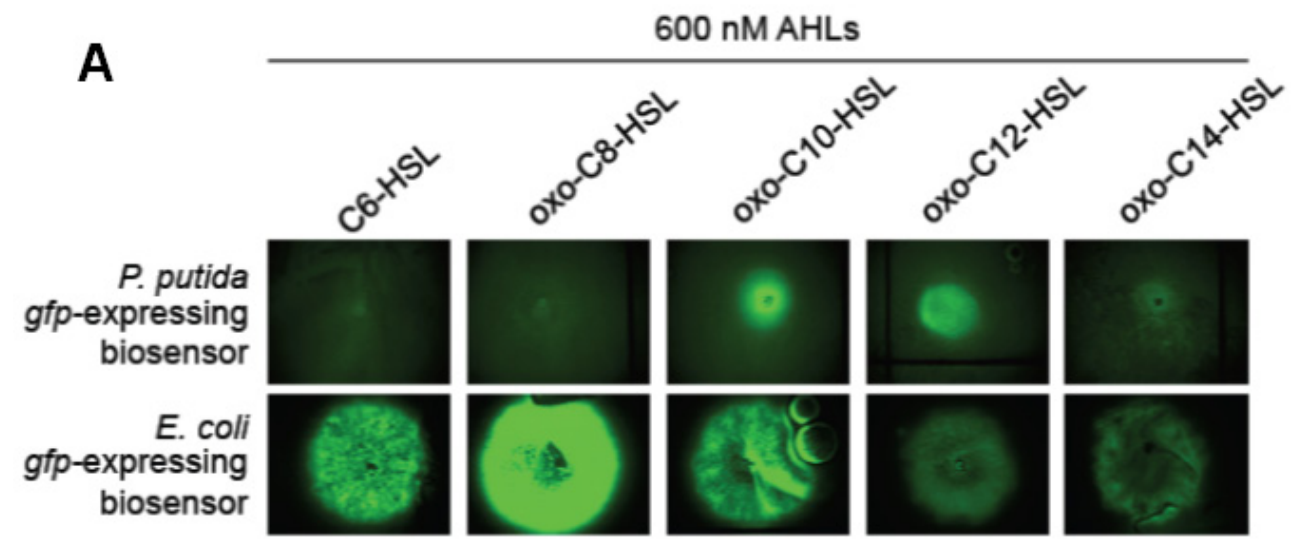

B

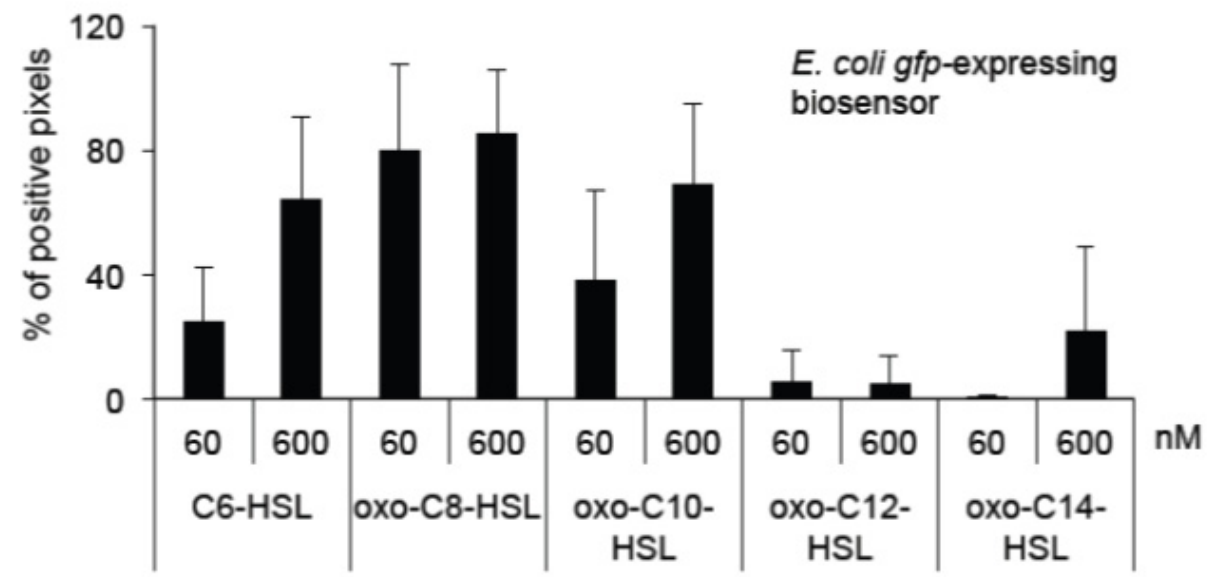


Figure S2. Production of AHL in R. etli strains used in this work. Different strains of $R$. etli were tested for AHL production using the bacterial biosensor E. coli MT102 (pJBA89) (A) AHLs produced by the indicated $R$. etli strains were extracted with $\mathrm{CHCl}_{3}$ and applied onto a lawn of the E. coli biosensor. The GFP signal was observed $2 \mathrm{~h}$ thereafter. $R$. etli attM $\left(\mathrm{Km}^{R}\right)$ and $R$. etli $\mathrm{attM}^{+}\left(\mathrm{Gm}^{R}\right)$ are strains expressing the attM gene from A. tumefaciens coding for a lactonase; (B) Growth curves of the $R$. etli strains. Bacteria were grown in $5 \mathrm{~mL}$ culture with constant agitation $(100 \mathrm{rpm})$ at $21^{\circ} \mathrm{C}$. $\mathrm{OD}_{600 \mathrm{~nm}}$ was measured every $4 \mathrm{~h}$.

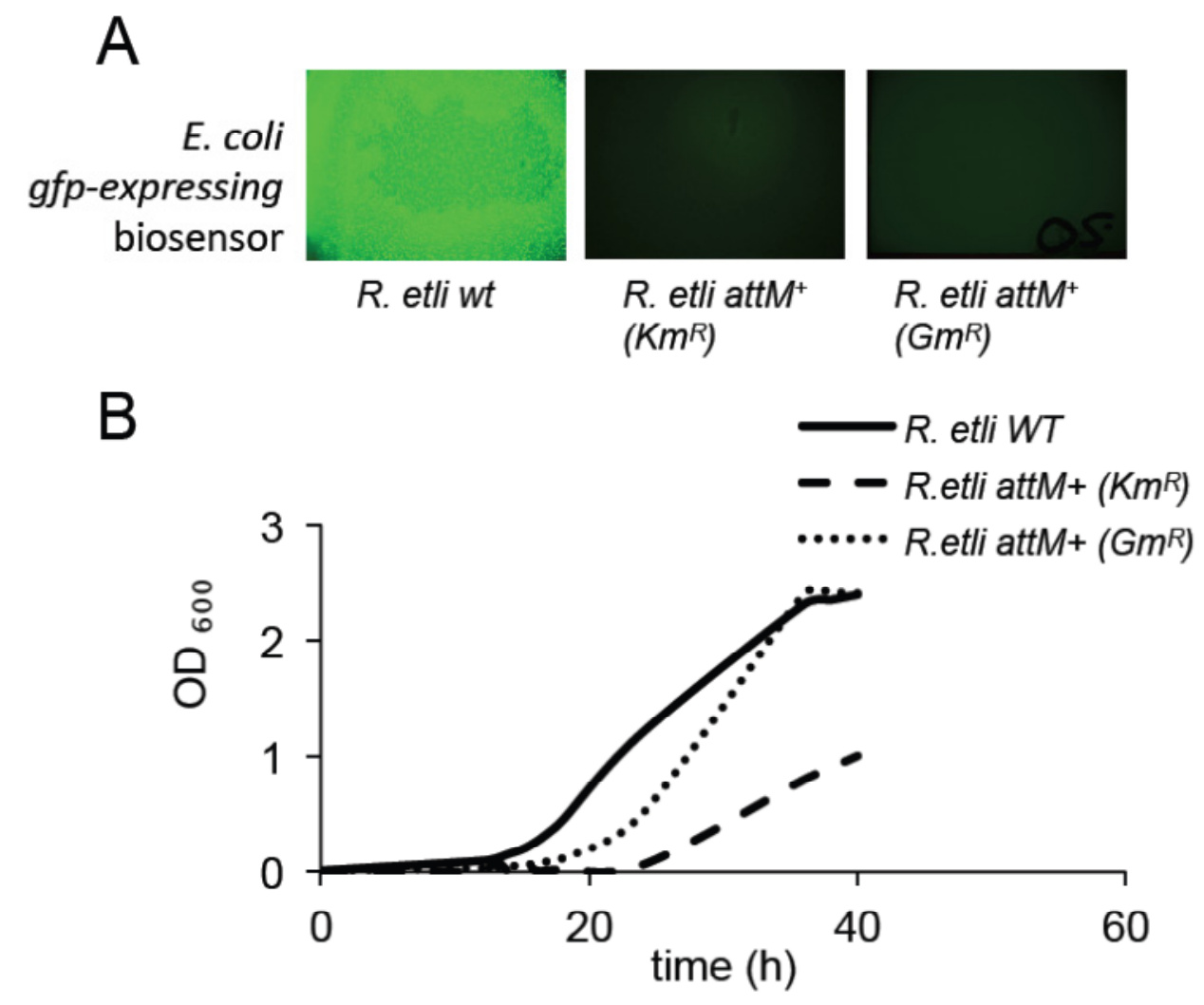


Figure S3. $R$. etli strains have no impact on Arabidopsis resistance towards $P$. syringae bacteria. Proliferation of the plant pathogenic P. syringae DC3000 (Pst) on plants pretreated with $\mathrm{MgCl}_{2}$ (control) or different $R$. etli strains, producing different quantities of oxo-C8-HSL. (A) Pst bacteria were infiltrated into Arabidopsis leaves. $\mathrm{OD}_{600 \mathrm{~nm}}=0.01$; (B) Arabidopsis plants were spray-inoculated with $P$ st bacterial solution, $\mathrm{OD}_{600 \mathrm{~nm}}=0.1$; (C) AHL production in $R$. etli strains used in the above experiments detected by the bacterial biosensor strain of $E$. coli. Student's $t$-test revealed no differences between the treatments at $p \geq 0.05$, as indicated by the letters in (A) and (B).

A

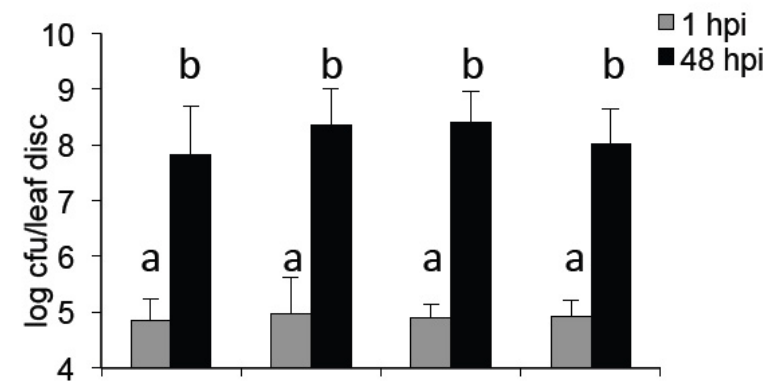

B

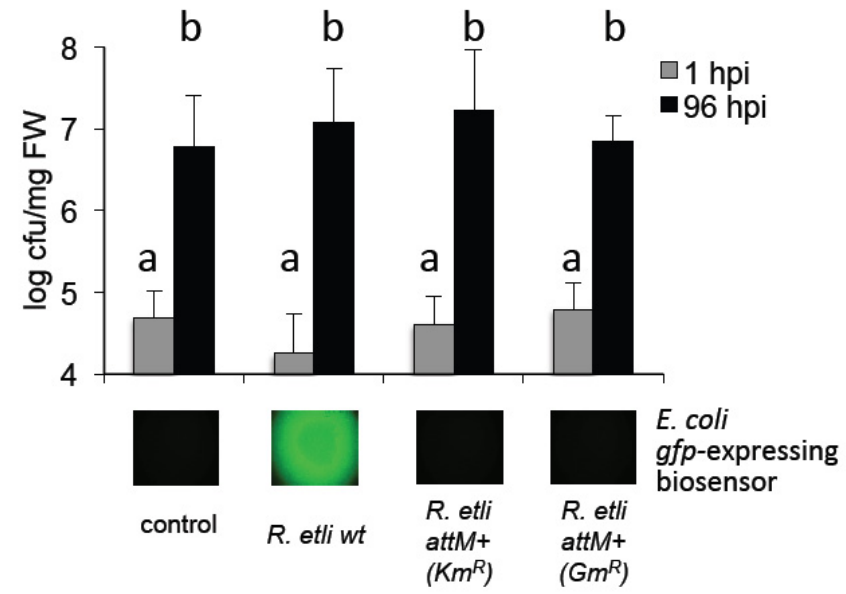

Figure S4. Growth of $S$. meliloti in the presence of Arabidopsis plants. Proliferation of $S$. melioti measured over the period of 2 days in medium with or without Arabidopsis seedlings.

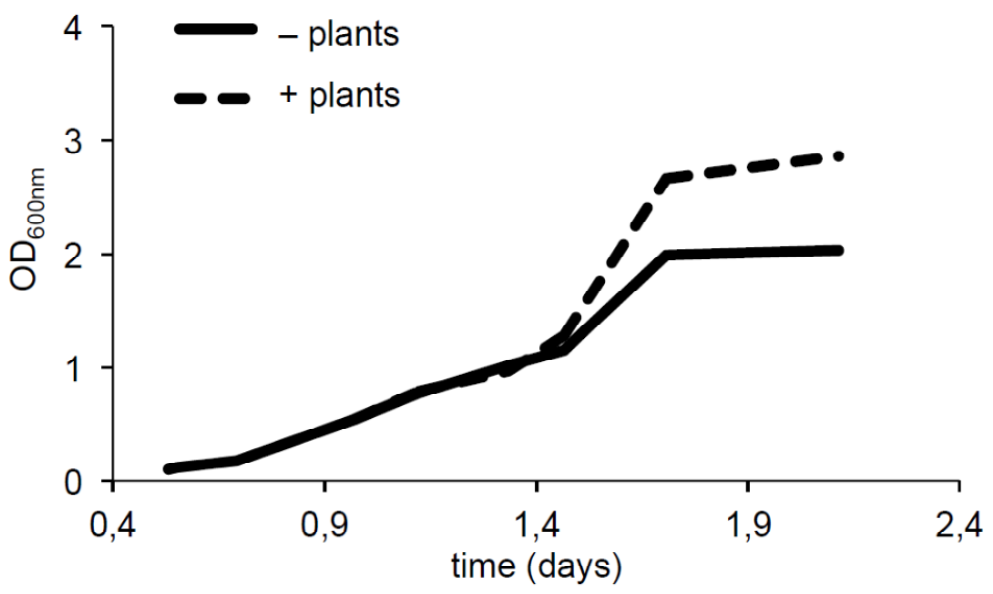




\section{References}

1. Fuqua, W.C.; Winans, S.C. A LuxR-LuxI type regulatory system activates Agrobacterium Ti plasmid conjugal transfer in the presence of a plant tumor metabolite. J. Bacteriol. 1994, 176, 2796-2806.

2. Kaplan, H.B.; Greenberg, E.P. Diffusion of autoinducer is involved in regulation of the Vibrio fischeri luminescence system. J. Bacteriol. 1985, 163, 1210-1214.

3. Schuhegger, R.; Ihring, A.; Gantner, S.; Bahnweg, G.; Knappe, C.; Vogg, G.; Hutzler, P.; Schmid, M.; van Breusegem, F.; Eberl, L.; et al. Induction of systemic resistance in tomato by $\mathrm{N}$-acyl-L-homoserine lactone-producing rhizosphere bacteria. Plant Cell Environ. 2006, 29, 909-918.

4. Gantner, S.; Schmid, M.; Durr, C.; Schuhegger, R.; Steidle, A.; Hutzler, P.; Langebartels, C.; Eberl, L.; Hartmann, A.; Dazzo, F.B. In situ quantitation of the spatial scale of calling distances and population density-independent $N$-acylhomoserine lactone-mediated communication by rhizobacteria colonized on plant roots. FEMS Microbiol. Ecol. 2006, 56, 188-194.

5. Pang, Y.; Liu, X.; Ma, Y.; Chernin, L.; Berg, G.; Gao, K. Induction of systemic resistance, root colonisation and biocontrol activities of the rhizospheric strain of Serratia plymuthica are dependent on $N$-acyl homoserine lactones. Eur. J. Plant Pathol. 2009, 124, 261-268.

6. Von Rad, U.; Klein, I.; Dobrev, P.I.; Kottova, J.; Zazimalova, E.; Fekete, A.; Hartmann, A.; Schmitt-Kopplin, P.; Durner, J. Response of Arabidopsis thaliana to $N$-hexanoyl-DL-homoserine-lactone, a bacterial quorum sensing molecule produced in the rhizosphere. Planta 2008, 229, 73-85.

7. Liu, F.; Bian, Z.; Jia, Z.; Zhao, Q.; Song, S. The GCR1 and GPA1 participate in promotion of Arabidopsis primary root elongation induced by $N$-Acyl-homoserine lactones, the bacterial quorum-sensing signals. Mol. Plant-Microbe Interact. 2012, 25, 677-683.

8. Schenk, S.T.; Stein, E.; Kogel, K.H.; Schikora, A. Arabidopsis growth and defense are modulated by bacterial quorum sensing molecules. Plant Signal. Behav. 2012, 7, 178-181.

9. Bai, X.; Todd, C.D.; Desikan, R.; Yang, Y.; Hu, X. N-3-oxo-decanoyl-L-homoserine-lactone activates auxin-induced adventitious root formation via hydrogen peroxide- and nitric oxide-dependent cyclic GMP signaling in mung bean. Plant Physiol. 2012, 158, 725-736.

10. Schikora, A.; Schenk, S.T.; Stein, E.; Molitor, A.; Zuccaro, A.; Kogel, K.H. N-acyl-homoserine lactone confers resistance towards biotrophic and hemibiotrophic pathogens via altered activation of AtMPK6. Plant Physiol. 2011, 157, 1407-1418.

11. Teplitski, M.; Eberhard, A.; Gronquist, M.R.; Gao, M.; Robinson, J.B.; Bauer, W.D. Chemical identification of $\mathrm{N}$-acyl homoserine lactone quorum-sensing signals produced by Sinorhizobium meliloti strains in defined medium. Arch. Microbiol. 2003, 180, 494-497.

12. Pellock, B.J.; Teplitski, M.; Boinay, R.P.; Bauer, W.D.; Walker, G.C. A LuxR homolog controls production of symbiotically active extracellular polysaccharide II by Sinorhizobium meliloti. J. Bacteriol. 2002, 184, 5067-5076.

13. Beringer, J.E. R factor transfer in Rhizobium leguminosarum. J. Gen. Microbiol. 1974, 84, 188-198.

14. Simon, R.; Priefer, U.; Pühler, A. A broad host range mobilization system for in vivo genetic engineering: Transposon mutagenesis in gram negative bacteria. Nat. Biotechnol. 1983, 1, 784-791.

15. McIntosh, M. University of Marburg, Marburg, Germany.Unpublished observation, 2013. 
16. Schikora, A. Justus Liebig University Giessen, Giessen, Germany. Unpublished oberservation, 2013.

17. Ortiz-Castro, R.; Martinez-Trujillo, M.; Lopez-Bucio, J. $N$-acyl-L-homoserine lactones: A class of bacterial quorum-sensing signals alter post-embryonic root development in Arabidopsis thaliana. Plant Cell Environ. 2008, 31, 1497-1509.

18. Perez-Montano, F.; Guasch-Vidal, B.; Gonzalez-Barroso, S.; Lopez-Baena, F.J.; Cubo, T.; Ollero, F.J.; Gil-Serrano, A.M.; Rodriguez-Carvajal, M.A.; Bellogin, R.A.; Espuny, M.R. Nodulation-gene-inducing flavonoids increase overall production of autoinducers and expression of $\mathrm{N}$-acyl homoserine lactone synthesis genes in Rhizobia. Res. Microbiol. 2011, 162, 715-723.

19. Garcia-Fraile, P.; Carro, L.; Robledo, M.; Ramirez-Bahena, M.H.; Flores-Felix, J.D.; Fernandez, M.T.; Mateos, P.F.; Rivas, R.; Igual, J.M.; Martinez-Molina, E.; et al. Rhizobium promotes non-legumes growth and quality in several production steps: Towards a biofertilization of edible raw vegetables healthy for humans. PLoS One 2012, 7, e38122.

20. Marketon, M.M.; Gonzalez, J.E. Identification of two quorum-sensing systems in Sinorhizobium meliloti. J. Bacteriol. 2002, 184, 3466-3475.

21. Pozo, M.J.; van der Ent, S.; van Loon, L.C.; Pieterse, C.M. Transcription factor MYC2 is involved in priming for enhanced defense during rhizobacteria-induced systemic resistance in Arabidopsis thaliana. New Phytol. 2008, 180, 511-523.

22. Van der Ent, S.; Verhagen, B.W.; van Doorn, R.; Bakker, D.; Verlaan, M.G.; Pel, M.J.; Joosten, R.G.; Proveniers, M.C.; van Loon, L.C.; Ton, J.; et al. MYB72 is required in early signaling steps of rhizobacteria-induced systemic resistance in Arabidopsis. Plant Physiol. 2008, 146, 1293-1304.

23. Gurich, N.; Gonzalez, J.E. Role of quorum sensing in Sinorhizobium meliloti-Alfalfa symbiosis. J. Bacteriol. 2009, 191, 4372-4382.

24. Mathesius, U.; Mulders, S.; Gao, M.; Teplitski, M.; Caetano-Anolles, G.; Rolfe, B.G.; Bauer, W.D. Extensive and specific responses of a eukaryote to bacterial quorum-sensing signals. Proc. Natl. Acad. Sci. USA 2003, 100, 1444-1449.

25. Hartmann, A.; Schikora, A. Quorum sensing of bacteria and trans-kingdom interactions of $N$-acyl homoserine lactones with eukaryotes. J. Chem. Ecol. 2012, 38, 704-713.

26. Marketon, M.M.; Gronquist, M.R.; Eberhard, A.; Gonzalez, J.E. Characterization of the Sinorhizobium meliloti $\operatorname{sinR} / \operatorname{sinI}$ locus and the production of novel $N$-acyl homoserine lactones. J. Bacteriol. 2002, 184, 5686-5695.

27. Gao, M.; Chen, H.; Eberhard, A.; Gronquist, M.R.; Robinson, J.B.; Rolfe, B.G.; Bauer, W.D. sinI- and expR-dependent quorum sensing in Sinorhizobium meliloti. J. Bacteriol. 2005, 187, 7931-7944.

28. Eberl, L.; Winson, M.K.; Sternberg, C.; Stewart, G.S.A.B.; Christiansen, G.; Chhabra, S.R.; Bycroft, B.; Williams, P.; Molin, S.; Givskov, M. Involvement of $N$-acyl-L-homoserine lactone autoinducers in controlling the multicellular behaviour of Serratia liquefaciens. Mol. Microbiol. 1996, 20, 127-136.

29. Schwachtje, J.; Karojet, S.; Thormahlen, I.; Bernholz, C.; Kunz, S.; Brouwer, S.; Schwochow, M.; Kohl, K.; van Dongen, J.T. A naturally associated rhizobacterium of Arabidopsis thaliana induces a starvation-like transcriptional response while promoting growth. PLoS One 2011, 6, e29382. 
30. Hense, B.A.; Muller, J.; Kuttler, C.; Hartmann, A. Spatial heterogeneity of autoinducer regulation systems. Sensors 2012, 12, 4156-4171.

31. Teplitski, M.; Chen, H.; Rajamani, S.; Gao, M.; Merighi, M.; Sayre, R.T.; Robinson, J.B.; Rolfe, B.G.; Bauer, W.D. Chlamydomonas reinhardtii secretes compounds that mimic bacterial signals and interfere with quorum sensing regulation in bacteria. Plant Physiol. 2004, 134, 137-146.

32. Gao, M.; Teplitski, M.; Robinson, J.B.; Bauer, W.D. Production of substances by Medicago truncatula that affect bacterial quorum sensing. Mol. Plant-Microbe Interact. 2003, 16, 827-834.

33. Perez-Montano, F.; Jimenez-Guerrero, I.; Sanchez-Matamoros, R.C.; Lopez-Baena, F.J.; Ollero, F.J.; Rodriguez-Carvajal, M.A.; Bellogin, R.A.; Espuny, M.R. Rice and bean AHL-mimic quorum-sensing signals specifically interfere with the capacity to form biofilms by plant-associated bacteria. Res. Microbiol. 2013, doi:10.1016/j.bbr.2011.03.031.

34. Manefield, M.; Rasmussen, T.B.; Henzter, M.; Andersen, J.B.; Steinberg, P.; Kjelleberg, S.; Givskov, M. Halogenated furanones inhibit quorum sensing through accelerated LuxR turnover. Microbiology 2002, 148, 1119-1127.

35. Keshavan, N.D.; Chowdhary, P.K.; Haines, D.C.; Gonzalez, J.E. L-Canavanine made by Medicago sativa interferes with quorum sensing in Sinorhizobium meliloti. J. Bacteriol. 2005, 187, 8427-8436.

36. Vandeputte, O.M.; Kiendrebeogo, M.; Rajaonson, S.; Diallo, B.; Mol, A.; El Jaziri, M.; Baucher, M. Identification of catechin as one of the flavonoids from Combretum albiflorum bark extract that reduces the production of quorum-sensing-controlled virulence factors in Pseudomonas aeruginosa PAO1. Appl. Environ. Microbiol. 2010, 76, 243-253.

37. Kovach, M.E.; Phillips, R.W.; Elzer, P.H.; Roop, R.M., II; Peterson, K.M. pBBR1MCS: A broad-host-range cloning vector. BioTechniques 1994, 16, 800-802.

38. Andersen, J.B.; Heydorn, A.; Hentzer, M.; Eberl, L.; Geisenberger, O.; Christensen, B.B.; Molin, S.; Givskov, M. gfp-based N-Acyl homoserine-lactone sensor systems for detection of bacterial communication. Appl. Environ. Microbiol. 2001, 67, 575-585.

39. Steidle, A.; Sigl, K.; Schuhegger, R.; Ihring, A.; Schmid, M.; Gantner, S.; Stoffels, M.; Riedel, K.; Givskov, M.; Hartmann, A.; et al. Visualization of $N$-acylhomoserine lactone-mediated cell-cell communication between bacteria colonizing the tomato Rhizosphere. Appl. Environ. Microbiol. 2001, 67, 5761-5770.

40. Schikora, M.; Hage, M.; Ruthotto, E.; Wild, K. A Convex Formulation for Color Image Segmentation in the Context of Passive Emitter Localization, Information Fusion, 2009, In Proceedings of the 12th International Conference on Information Fusion, 2009, FUSION 2009, Seattle, WA, USA, 6-9 July 2009; pp. 1424-1431.

(C) 2013 by the authors; licensee MDPI, Basel, Switzerland. This article is an open access article distributed under the terms and conditions of the Creative Commons Attribution license (http://creativecommons.org/licenses/by/3.0/). 